\title{
ATOMISTIC PREDICTION OF PLANE STRESS BEHAVIOR OF GLASSY THERMOSETS
}

\author{
James C. Moller ${ }^{1}$, Gary S. Kedziora ${ }^{2}$, Stephen A. Barr ${ }^{3}$, Timothy D. Breitzman ${ }^{3}$, Rajiv J. Berry ${ }^{3}$ \\ 1. Miami University \\ Department of Mechanical and Manufacturing Engineering \\ Oxford, $\mathrm{OH} 45056$ \\ 2. Engility Corporation \\ Air Force Research Laboratory \\ Wright-Patterson AFB, OH 45433 \\ 3. Air Force Research Laboratory \\ Materials \& Manufacturing Directorate \\ Wright-Patterson AFB, OH 45433
}

\begin{abstract}
When any covalent bond energy reached a threshold indicative of incipient bond scission, the simulation was stopped. This method was used to examine the responses of three highlycrosslinked epoxy systems. Systems were large enough to include 110 to 480 crosslink sites. Both elastic and yield properties show good agreement with the experiments of others. Continuum yield theories commonly applied to polymers are compared with the data. A DruckerPrager pressure-dependent yield function applied best in the second and third quadrants of the $\sigma_{1} \sigma_{2}$ domain. In the first quadrant, however, data more closely match a normal-stress-yielding criterion. In biaxial tension and simple tension, plastic behavior and large growth in nanoporosity were observed. Ductility was lowest in simple tension and biaxial compression. In simple tension, the bonds at crosslink sites and in ether linkages were the most highly strained whereas carbon-carbon backbone bonds between phenyl groups were highly strained in other cases. When system energy at imminent bond rupture was divided on a per-atom basis, consistency with Peterlin's theory for molecular rupture was found.
\end{abstract}

\section{Introduction}

Characterization of plane stress behavior of a given material is of great utility. For example, knowledge of yield strength and plastic flow behavior in plane stress can be used to estimate the plastic zone size and shape ahead of a crack tip and therefore support the estimation of material toughness. When plane stress properties can be expressed in terms of a small number of parameters, the results can be readily embedded in numerical simulations and therefore enable prediction of stress and deformation in objects having more complicated shapes. When 
plane stress behavior is derived from atomistic interactions, fundamental insight is provided into the ways atomic and molecular structural quantities affect bulk response.

Numerous yield theories have been put forth to provide a generalized yield threshold or lower bound. These are aimed at capturing the general continuum behavior in terms of underlying key independent variables (e.g., strain energy) or a small number of measured properties (e.g., uniaxial tensile yield strength). The classic theories provide, in particular, yield thresholds in terms of invariants (e.g., [1, 2, 3, 4, 5]), shear stress (e.g., [6]), or combined shear and normal stress thresholds (e.g., [7]).

While these theories are often appropriately applied to describe polycrystalline equiaxed metals and basic structural materials (e.g., concrete), they are not as robust for characterizing polymers. While some experiments match some of the afore-mentioned criteria well [8,9], others indicated distinctly different mechanisms at yield according to load case [10, 11, 12]. In the latter experiments, the materials showed distinctly lower strength in load cases which included positive dilatation. This weakness was inconsistent with the strength in load cases where distortion or negative dilatation were predominant in that the loci of data points among these case types followed differing functional dependencies. Where thermoplastics were investigated, it was generally found that the weakness was associated with crazing whereas shear bands occurred in other load cases. When thermosets were investigated, there were instances where the material would rapidly proceed to fracture whereas it would display measureable ductility in others [8,12]. In some instances, the onset of these differing responses could be matched with threshold values of stress or strain invariants [11]. Some attempts have been made to explain the origins of these using continuum-mechanics-based arguments [13] Additional yield criteria based on observations from experiments have been developed [14, 15, $5816,17,18]$.

Fundamental insight is provided when stress responses are derived from smaller-scale (e.g., molecular) behaviors. Direct connections among chemical structure and bulk behaviors can be sought to substantiate physical bases for the dependencies that are observed in experiments or included in theories. Some theories for the onset of polymer plastic flow derive from fundamental considerations of molecular mechanical and stochastic behavior. Yannas and Lunn [19] explored and compared the effects of inter- and intra-molecular forces on the energy barriers to backbone dihedral rotation and anticipated that inter-molecular effects would be significant below $T_{g}$. Robertson [20] considered the application of shear stress and the resulting probability for backbone conformation changes. He concluded that the system volume would increase and a liquid-like state would exist. Bowden and Raha [21] envisioned strain fields similar to dislocation loops in crystalline metals associated with the rearrangement of molecular chains as shear stress is applied and fit their model to data from experiments. Joseph [22] aimed to provide a model which would display both yield and relaxation transition behaviors based on linearly elastic elements and a potential with multiple barriers. Only systems undergoing pure shear were considered. Argon [23] associated strain with the production of backbone kink pairs and derived the energy barrier due to intermolecular interactions. The theory was tested by checking the fit to compressive and tensile yield measurements for numerous polymers over wide ranges of temperature and strain rate $[23,24]$. While it was 
asserted that a pressure-modified von Mises yield criterion would apply to generalized plane stress based on selected data [24], the explanation of experimental results associated with void nucleation - and decidedly differing from von-Mises-like functions - was not directly related to the kink pair mechanism.

Experiments have been performed to ascertain the molecular behavior associated with mechanical loading. Some monitored material dilatation by measuring variation of free volume size and distribution due to loading [25, 26, 27, 28, 29, 30, 31]. Others examined distortion by inferring chain reorientation and changes in side group concentration from infrared spectra [32, $33,34]$. At the point of incipient rupture, still others used a combination of techniques to identify correlations among bond scissions, radical concentration, and crack nucleation [35, 36, 37, 38]. The latter results indicate molecular scission generally leads to a cascade of mechano-chemical reactions and the occurrence of micro-scale voids.

We know of no broader thorough exploration of polymer mechanical behavior derived from atomistic simulation. Atomistic simulations of mechanical deformation of glassy polymers thus far have principally involved uniaxial or pure shear loading $[39,40,41,42,43,44,45,46,47$, 48] $[49,50,51,52,53,54,55,56]$. Such an investigation is important for the sake of demonstrating the relevance of molecular dynamics results for properties prediction at larger length scales as well as for providing additional insight into the molecular bases for yield, flow, and rupture. While atomistic simulation has been used to predict the yield surface of metallic glass [57], the interatomic potentials appropriate for polymers are more varied and complex. In one instance, a yield locus was asserted based on atomistic simulations of polymers in pure tension and pure compression [58]. As will be shown, however, a thorough investigation in each quadrant of the $\sigma_{1}-\sigma_{2}$ domain shows the effect of hydrostatic stress on yield. A method for applying generalized plane stress to an atomistic system is presented as well as an interpretation of the results for a set of thermoset formulations. The principal independent variable among the formulations is crosslink density. Once the methods and algorithm are described, results are presented and compared with physical experiments of other researchers. System behaviors are expressed in terms of stress and strain invariants. Further, underlying molecular behaviors associated with yield and rupture are elicited.

\section{Methods}

\section{Plane stress application}

\section{Algorithm}

An algorithm was designed to apply a succession of stress states in a sequence of molecular dynamics simulations such that a set proportion of in-plane stresses as well as a target equivalent strain rate were maintained. The stress state is given by 


$$
\boldsymbol{\sigma}=\left[\begin{array}{ccc}
\sigma_{x x} & 0 & 0 \\
0 & \sigma_{y y} & 0 \\
0 & 0 & 0
\end{array}\right]
$$

113 In what follows, an angle $\theta$ will be associated with the stress state. This is depicted in Figure 1.

114 Depending on their relative magnitudes, $\sigma_{x x}$ and $\sigma_{y y}$ correspond with principal stresses $\sigma_{1}$ and $115 \sigma_{2}$

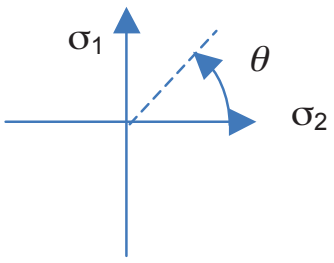

Figure 1. Definition of the angle associated with each applied stress state. As deformation proceeds, the proportion of principal stresses is maintained.

A definition of the stress tensor in terms of quantities germane to molecular dynamics is given by

$$
\boldsymbol{\sigma}=-\frac{1}{V}\left\langle\sum_{i=1}^{N} m_{i} \mathbf{v}_{i} \otimes \mathbf{v}_{i}+\mathbf{W}\left(\mathbf{r}^{N}\right)\right\rangle
$$

where \langle\rangle denotes an ensemble average, $V$ is the volume enclosing the system, $N$ is the number of particles, $m$ is particle mass, $\mathbf{v}$ is particle velocity, $\mathbf{r}$ is particle location, and $\mathbf{W}$ is the virial tensor. Thompson, et al. [59] derived descriptions of the virial tensor appropriate for periodic conditions. The atom-cell form of $\mathbf{W}$ is given by

$$
\mathbf{W}\left(\mathbf{r}^{N}\right)=\sum_{i=1}^{N} \mathbf{r}_{i} \otimes \mathbf{F}_{i}+\sum_{\mathbf{n} \in Z^{3}} \mathbf{H n} \otimes \sum_{i=1}^{N} \mathbf{F}_{i \mathbf{n}}^{\prime}
$$

where $\mathbf{H}$ is a matrix of the periodic cell vectors, $\mathbf{n}$ is an integer vector for the offset to the respective periodic image, and $\mathbf{F}$ is the force acting on the particle. $\mathbf{F}^{\prime}$ is the partial force on the particle due to groups associated with the local cell. F can originate from both pairwise and multibody interactions.

Plane stress is attained by setting the respective target values for stress tensor terms of an isobaric-isothermal ensemble. In order to maintain a steady strain rate, plane stress states are applied in a succession of brief periods. At the end of each, equivalent strain (based on cell dimensions) is compared against the target value for the corresponding total elapsed time. Equivalent strain is given by 


$$
\varepsilon_{\text {equ }}=\left[\frac{1}{\sqrt{2}} \sqrt{\left(\varepsilon_{x}-\varepsilon_{y}\right)^{\prime}+\left(\varepsilon_{y}-\varepsilon_{z}\right)+\left(\varepsilon_{z}-\varepsilon_{x}\right)^{2}+6\left(\varepsilon_{x y}^{2}+\varepsilon_{y z}^{2}+\varepsilon_{z x}^{2}\right)}\right]
$$

The applied in-plane stress values for the subsequent period are incremented from the previous interval in proportion to any error in equivalent strain as well as the rate-of-change in strain error. For tension or compression in the $x$ direction, this is given by

$$
\Delta \sigma_{x x}=C_{1}\left(\hat{\varepsilon}_{e q u}-\varepsilon_{e q u}\right)+C_{2} \frac{\Delta\left(\hat{\varepsilon}_{e q u}-\varepsilon_{e q u}\right)}{\Delta t}, \Delta \sigma_{y y}=0
$$

and

$$
\Delta \sigma_{x x}=C \Delta \sigma_{y y}=C_{1}\left(\hat{\varepsilon}_{e q u}-\varepsilon_{e q u}\right)+C_{2} \frac{\Delta\left(\hat{\varepsilon}_{e q u}-\varepsilon_{\text {equ }}\right)}{\Delta t}
$$

otherwise. $\hat{\varepsilon}_{\text {equ }}$ is the target equivalent strain.

Stresses therefore increase, decrease, or remain constant according to whether the material is hardening, softening, or perfectly plastic over the given time period. The algorithm flow is provided in Figure 2.

In addition to calculating strain error, covalent bond lengths are logged after each dynamics interval. If any are found to have reached a length deemed indicative of incipient rupture, the simulation is terminated. Simulation was not continued beyond the first indication of bond rupture because the experimental results of Zhurkhov, et al. [36] provide evidence that bond rupture leads to a cascade of mechano-chemical reactions and a corresponding appearance of large voids. A critical energy level is therefore associated with each covalent bond type. For example, if a harmonic bond stretching potential is given by

$$
E=K\left(r-r_{o}\right)^{2},
$$

a critical bond length associated with a critical energy level is then

$$
r_{\text {crit }}=\sqrt{\frac{E_{c r i t}}{K}}+r_{o} \text {. }
$$

Values for $E_{\text {crit }}$ came from the Morse bond potentials of the corresponding covalent atom pair. This potential is given by

$$
E=D\left[1-e^{-\alpha\left(r-r_{o}\right)}\right]
$$

where $D$ is the energy difference between $r=r_{o}$ and $r=\infty$. This is treated as $E_{c r i t}$. 
Input: Equilibrated system, target stress proportion, equivalent strain rate, estimated modulus

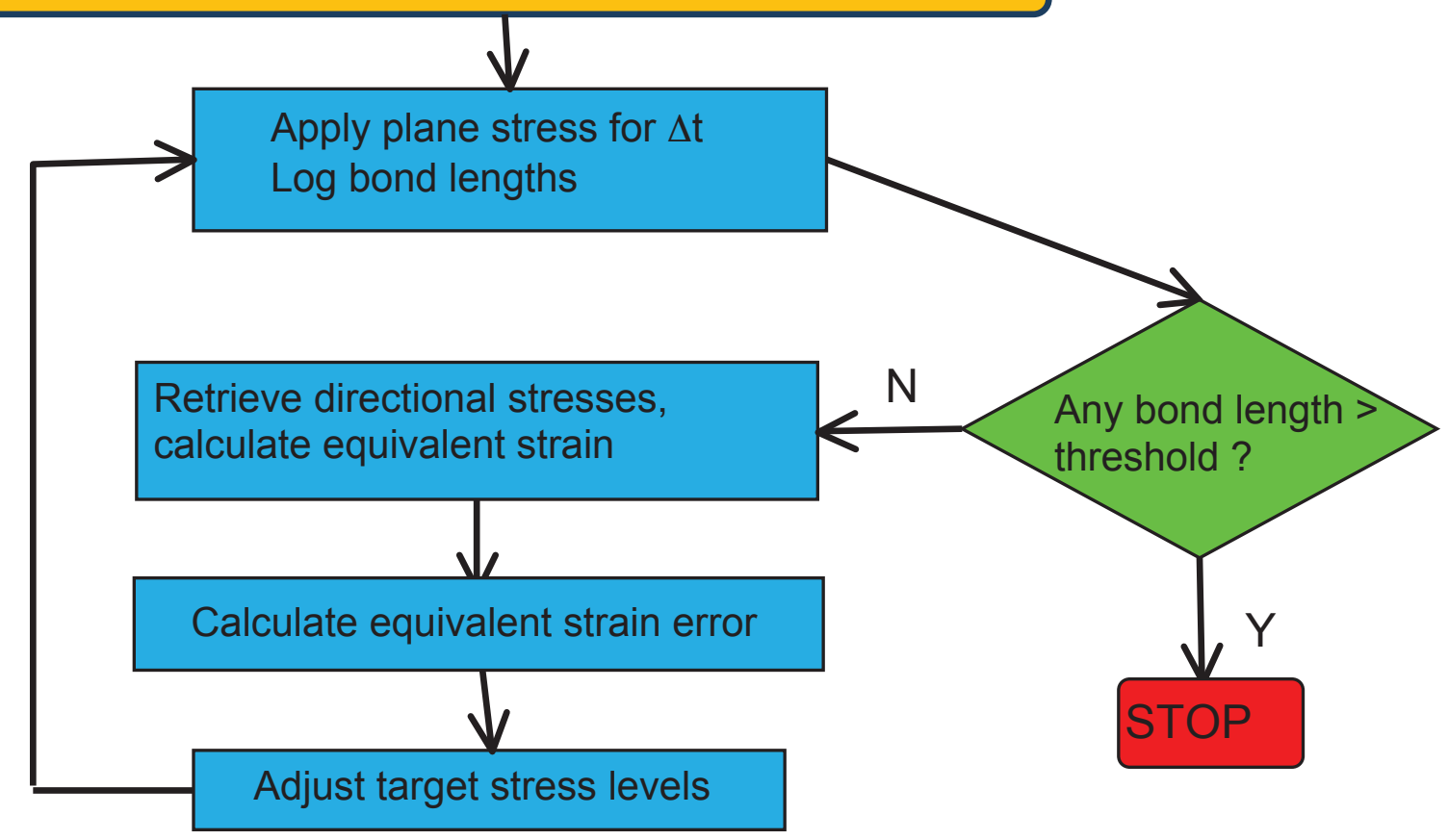

Figure 2. The flow chart for application of plane stress at a constant equivalent strain rate.

\section{Molecular systems}

The molecular systems investigated began as stoichiometric mixtures of monodisperse diglycidyl ether of bisphenol A (DGEBA) resin and methylene diamine (MDA) prepolymers. The principal difference among the systems studied was DGEBA chain length (and therefore cured cross-link density). The molecular structures are shown in Figure 3. These formulations have been carefully studied experimentally to determine their toughness and associated crack tip deformation in plane stress [60]. The systems are summarized in Table 1.

We are exploring whether, at length scales approaching an order of magnitude larger than the mean distance between crosslink sites, the system mechanical behavior takes on a character consistent with observed macro-scale observations.

The systems created represent essentially uniformly crosslinked continuous matrices. While there have been observations of nano-scale inhomogeneity [61] evolving during curing, there are also combinations of reactants and curing conditions that result in a homogeneous network. As discussed by Pascault, et al. [62], if a stoichiometric two-part system with simple functionality (such as those examined here) is thoroughly blended prior to crosslinking and reactions proceed in step-wise (rather than chain-wise) sense, the cured system is highly homogeneous.

Research has shown that mechanical properties are functions of $M_{c}$, the mean molecular weight of a strand between crosslinks or entanglements [63, 60, 64, 65, 66]. A closely-related quantity is the cross-link density. The crosslink densities here are similar to those reported for 
the same systems [60] in which the values ranged from $135 \times 10^{25} \mathrm{~m}^{-3}$ (for $\mathrm{n}=0$ ) to $33.5 \times 10^{25} \mathrm{~m}^{-}$ ${ }^{3}$ (for $n=5$ ). The system responses are largely governed by the collective responses of the molecular strands between crosslink sites. The number of strands scales approximately with the number of original molecules (which range from 171 to 738 here). Each strand, then, is a fraction of a percent to the entire system.

a)

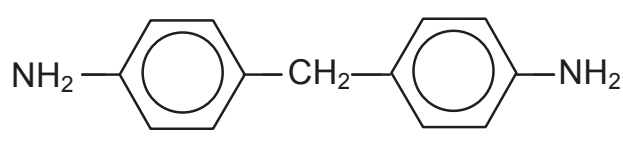

b)
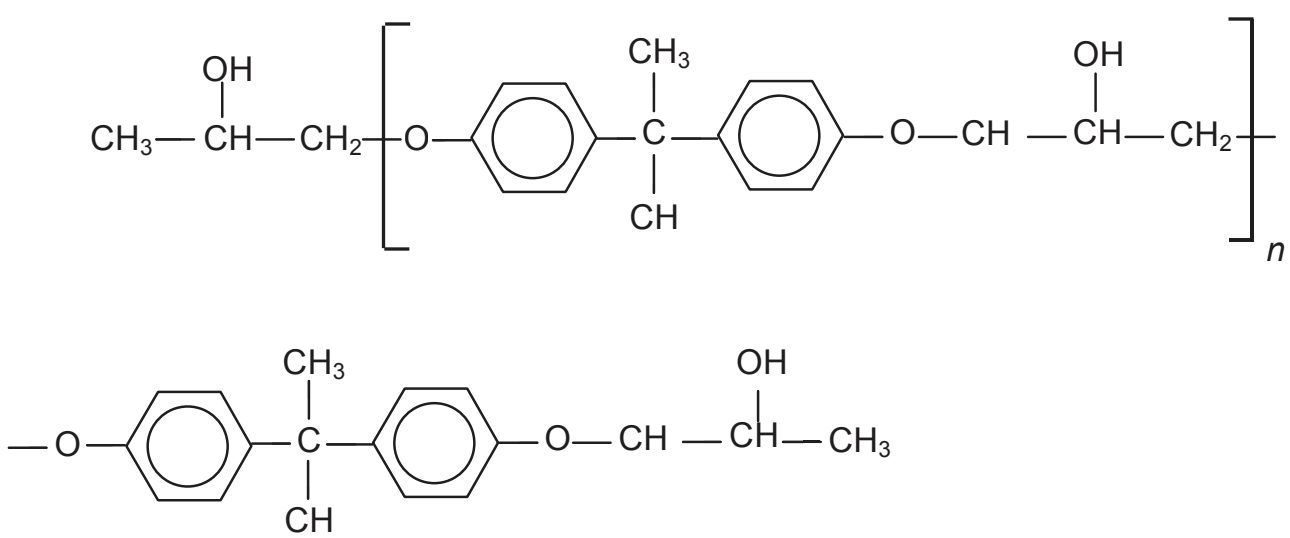

Figure 3. Molecular structures of MDA (a) and DGEBA (b). The epoxide group of DGEBA is shown replaced with methyl and hydroxyl groups. During crosslinking simulation, methyl carbon and amine nitrogen atoms become covalently bonded. For the systems investigated, $n$ $=0,3$, and 5 .

Systems were cross-linked during molecular dynamics simulation by the method of Rigby, et al. [67]. A proximity-based criterion is used to alter covalent bonds among open epoxide and amine groups to represent amine addition reactions to form secondary and tertiary amines. During dynamics, the proximities among potentially-reactive groups are measured. When any fall within a set range, one hydrogen atom is removed from each group and a new $\mathrm{C}-\mathrm{N}$ covalent bond is created. Energy local to the new bond is minimized before proceeding with subsequent dynamics. The crosslinking algorithm forms new bonds between crosslink sites based on a succession of searches of increasing radius with dynamics to allow relaxation and diffusion. If a new bond is formed which spears a phenyl ring, the system energy increase would be dramatically larger than that for the formation of a proper cross-link. The algorithm includes an energy increase threshold value. If system energy from one crosslink cycle to the next rises by more than this value, the newly-formed bond is rejected. The molecular system database reverts to that prior to the crosslinking attempt and additional dynamics is performed prior to attempting to form another bond. Curing was performed at $473 \mathrm{~K}$. Pressure was held at $0.5 \mathrm{GPa}$ until the cross-linked extent reached $55 \%$. Pressure then incrementally decreased as additional crosslinking occurred. Crosslink extent is defined as the portion of the original amine $\mathrm{N}-\mathrm{H}$ 
bonds which had reacted. Crosslinked systems were then equilibrated to one atmosphere and $298 \mathrm{~K}$. The criterion used to deem the system as being in equilibrium was that mean system volume (as averaged over $2.5 \mathrm{ps}$ ) changed by $0.25 \%$ or less over a span of 25 ps. Once crosslinked, the systems were once again equilibrated at elevated temperature and then cooled to the temperature for mechanical testing $(298 \mathrm{~K})$ and re-equilibrated.

Because it is well-known that thermoset glassy properties are a function of the difference between the test and glass transition temperatures [62], results are provided to confirm the systems were in a glassy state. Plots of specific volume versus temperature during cooling indicated glass transition temperatures of ca. 455 to $465 \mathrm{~K}$ (see Figure 4). While the simulated cooling rate is far greater than created in experiments, as discussed by Pascault, et al. [62] the activation energy associated with the characteristic time to reach equilibrium is very large for thermosets. As a result, the effect of cooling rate on the recorded glass transition temperature is weak. Lemay, et al, [63] found $T g=432 \mathrm{~K}$ for a short-chain $(n=0.14)$ DGEBA/MDA system. Levita, et al. [68] found Tg ranging from 378 to $433 \mathrm{~K}$ in DGEBA/DDS systems having the same range of crosslink density examined here.

Atom interactions were specified by the CVFF [69] force field. Harmonic bond potentials were used. Ewald summations were used for long-range interactions. Cutoffs for Lennard-Jones and Coulomb pair interactions were 12 and $16 \mathrm{~A}$, respectively.

Table 1. Summary of the molecular systems investigated.

\begin{tabular}{|c|c|c|c|c|c|c|c|c|}
\hline System & $\mathbf{n}$ & $\begin{array}{l}\text { MDA } \\
\text { molecules }\end{array}$ & $\begin{array}{l}\text { DGEBA } \\
\text { molecules }\end{array}$ & $\begin{array}{l}\text { Total } \\
\text { atoms }\end{array}$ & $\begin{array}{l}\text { Equilibrated } \\
\text { volume }\left(A^{3}\right)\end{array}$ & $\begin{array}{l}\text { Crosslink } \\
\text { extent }\end{array}$ & $\begin{array}{l}\text { Tertiary/secondary/ } \\
\text { primary amines }\end{array}$ & $\begin{array}{l}\text { Cross- } \\
\text { link } \\
\text { density } \\
\left(10^{\wedge} 25\right. \\
\left.m^{\wedge}(-3)\right)\end{array}$ \\
\hline $\mathrm{n} 0$ & 0 & 246 & 492 & 33210 & 343300 & 0.94 & $446 / 37 / 9$ & 123 \\
\hline n3 & 3 & 82 & 164 & 31242 & 333700 & 0.92 & $144 / 15 / 5$ & 40 \\
\hline n5 & 5 & 57 & 114 & 31065 & 334000 & 0.92 & $100 / 10 / 4$ & 27 \\
\hline
\end{tabular}




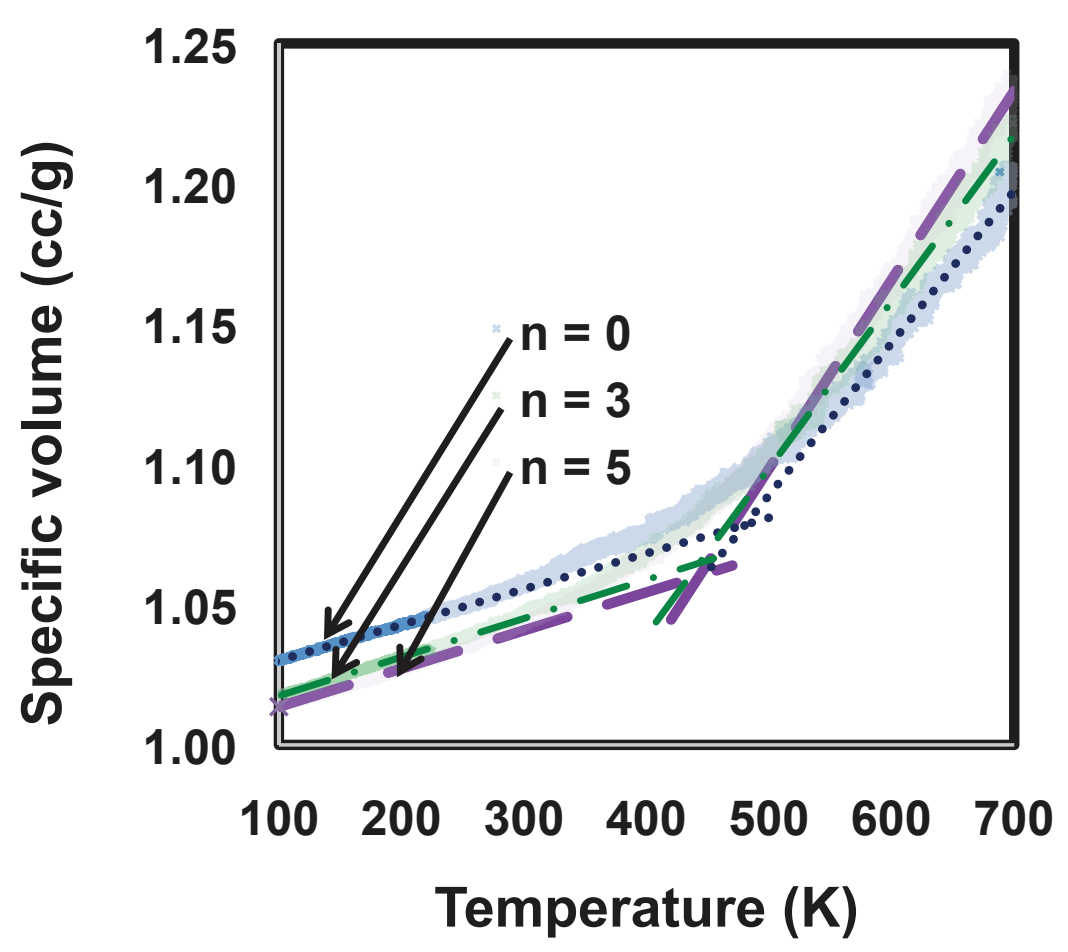

Figure 4. Plot of specific volume as a function of temperature for cooling from 698 to $98 \mathrm{~K}$ at a rate of $1.43 \times 10^{11} \mathrm{~K} / \mathrm{s}$.

254 Testing

255 The constants relating strain errors to the magnitude of the applied stress increment as well as 256 the barostat damping time constant were adjusted until reliably steady equivalent strain rate and 257 actual stress proportion values over the duration of each simulation were obtained. Example 258 histories are shown in Figure 5.

259 Table 2 provides the critical bond lengths and energies for the backbone bond types which, 260 when attained, would trigger the simulation end. The results of experiments have indicated 261 activations energies at a similar level [70]. LAMMPS [71] was used as the dynamics 262 environment and a Berendsen barostat applied. Equivalent strain rate was $10^{10} \mathrm{~s}^{-1}$. Sixty 263 different proportions and signs of in-plane stresses were applied and ranging from biaxial 264 tension to biaxial compression. 


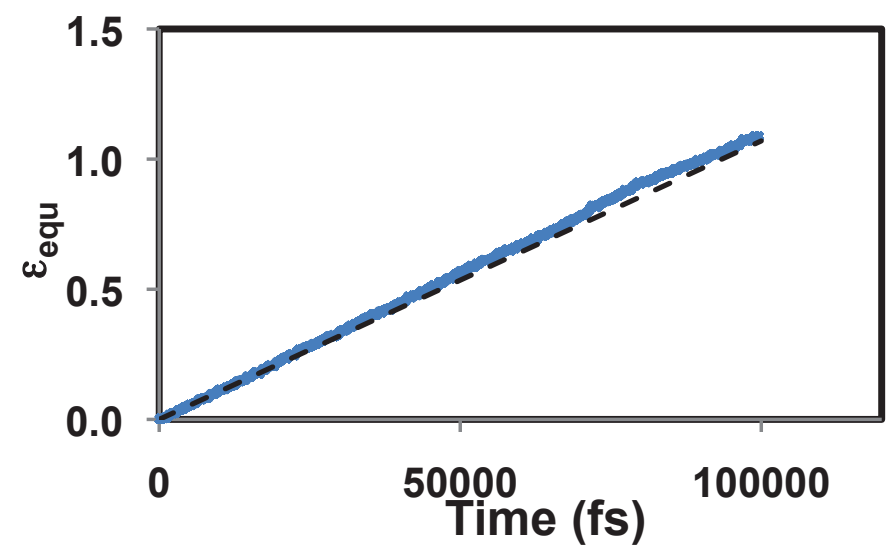

a)

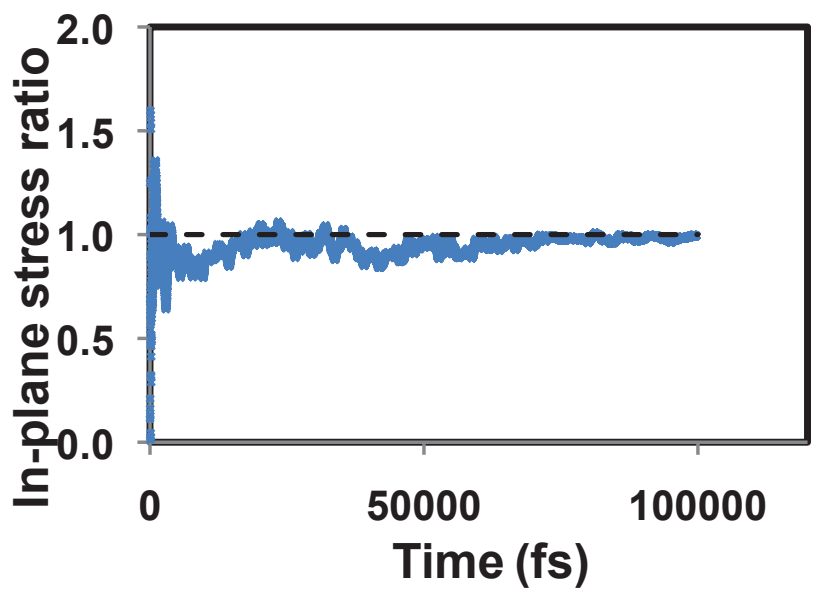

b)

Figure 5. Equivalent strain (a) and in-plane stress ratio (b) as a function of time for biaxial compression for system no. Results and target values are solid and dashed, respectively.

Table 2. Backbone CVFF atom pairs, harmonic potential parameters, Morse potential dissociation energy, and corresponding critical bond length.

\begin{tabular}{|c|c|c|c|c|c|}
$\begin{array}{c}\text { Atom } \\
\text { type }\end{array}$ & $\begin{array}{c}\text { Atom } \\
\text { type }\end{array}$ & $\begin{array}{c}\mathrm{K}(\mathrm{kcal} / \\
\left.\mathrm{mol} \mathrm{A}^{2}\right)\end{array}$ & $\mathrm{r}_{\mathrm{o}}(\mathrm{A})$ & $\begin{array}{c}\mathrm{D} \\
\text { (kcal/mol) }\end{array}$ & $\mathrm{r}_{\text {crit }}(\mathrm{A})$ \\
\hline $\mathrm{C}$ & $\mathrm{C}$ & 322.7158 & 1.526 & 88.0 & 2.048 \\
\hline $\mathrm{C}$ & $\mathrm{n}$ & 377.5752 & 1.460 & 72.0 & 1.897 \\
\hline $\mathrm{C}$ & $\mathrm{O}$ & 273.2000 & 1.425 & 68.3 & 1.925 \\
\hline
\end{tabular}




\section{Results and Discussion}

\section{General Behavior}

A broad initial comparison among the responses shows the predominant differences according to load case. Five cases (biaxial tension, tension, pure shear, compression, and biaxial compression) for system n0 were compared in terms of relations among stress and strain invariant-based quantities. These are mean stress (eqn. 13), equivalent stress (eqn. 14), the first strain tensor invariant - or volumetric dilatation - (eqn. 16), and equivalent strain (eqn. 17).

$$
\sigma_{m}=\frac{I_{1}}{3}=\frac{1}{3}\left(\sigma_{1}+\sigma_{2}+\sigma_{3}\right)
$$

$$
\sigma_{\text {equiv }}=\frac{1}{\sqrt{2}} \sqrt{\left(\sigma_{1}-\sigma_{2}\right)^{2}+\left(\sigma_{2}-\sigma_{3}\right)^{2}+\left(\sigma_{3}-\sigma_{1}\right)^{2}}=\sqrt{I_{1}^{2}-3 I_{2}}
$$

where

$$
I_{2}=\sigma_{1} \sigma_{2}+\sigma_{2} \sigma_{3}+\sigma_{3} \sigma_{1}
$$

where

$$
J_{1}=\varepsilon_{1}+\varepsilon_{2}+\varepsilon_{3}
$$

and where $I_{1}$ and $I_{2}$ are the first and second stress tensor invariants, $J_{1}$ and $J_{2}$ are the first and second strain tensor invariants, and stress and strain subscripts denote principal values.

Replicability of the behavior was examined by crosslinking and testing multiple systems. Each system was differently randomized when populating the cell with reacting molecules as well as when dynamics were performed to crosslink and equilibrate. The systems were the same in all other regards. Each was deformed in uniaxial tension. The results are shown in Figure 6 . The systems agree to within the stochastic scatter of the data until upper yield. Lower yield values The systems with a higher hardening rate showed higher strength and lower ductility. 


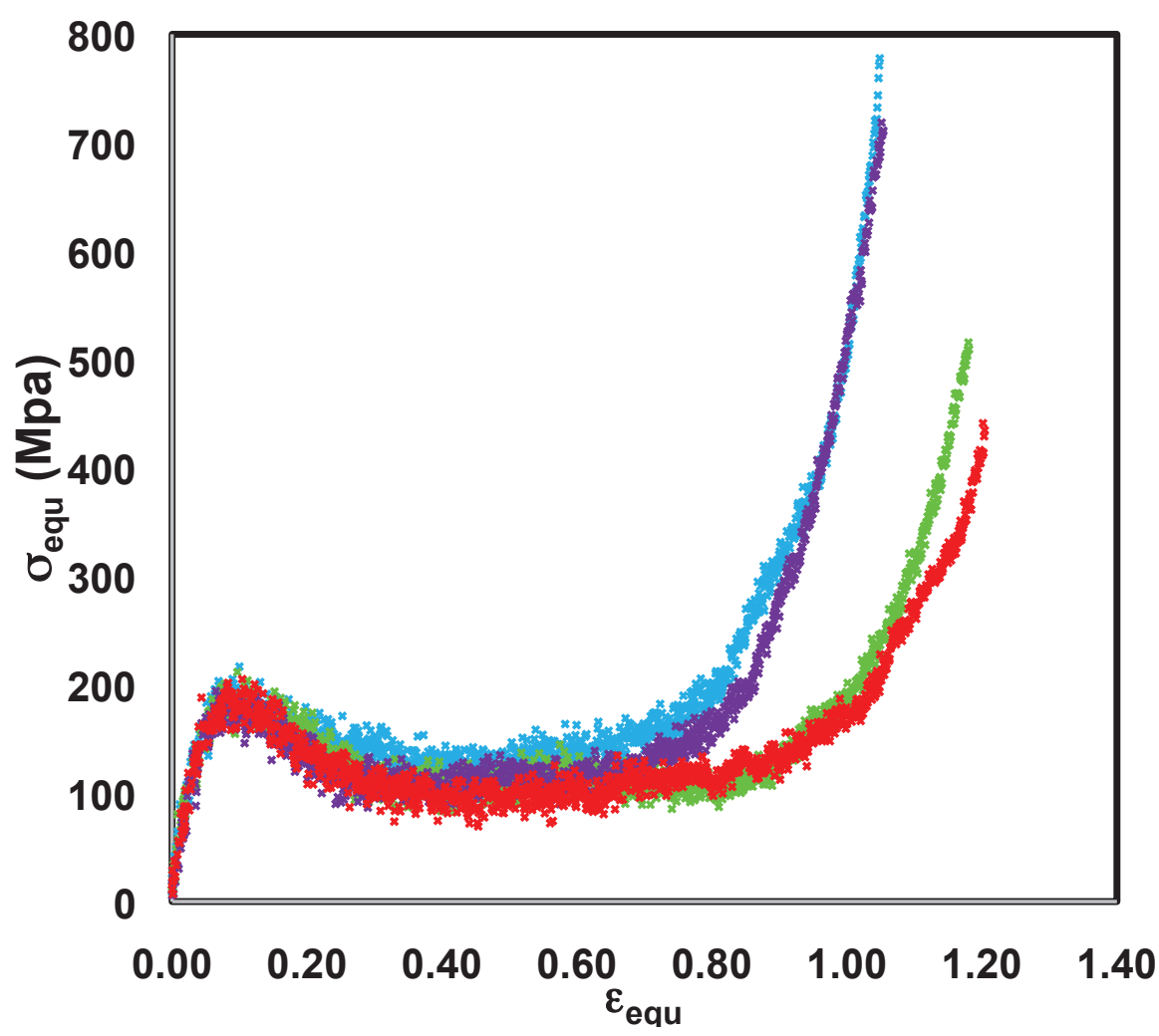

Figure 6. Equivalent stress-strain curves for four crosslinked systems. The systems have the same composition as system $\mathrm{n} 0$ and are cross-linked to $80 \%$.

When equivalent stress and strain scalar (quantities often associated with shear-associated deformation) are plotted with respect to each other, there are several distinguishing features. As shown in Figure 7, whereas tension and biaxial tension show an upper yield followed by an extended range of almost perfectly plastic flow, only hardening occurred in the other cases. The system was also distinctly stronger in compression cases than tension cases. Gerlach et al. also observed no strain softening in uniaxial compression tests of a thermoset resin over five decades of strain rate [72]. In experiments carefully controlled to create uniform stress and deformation of amine-cured DGEBA samples, Liang and Liechti [73] measured the large-strain mechanical response of in a variety of load cases. While post-yield softening and shear banding were found in tension, they also found perfectly plastic flow followed by hardening in pure compression and no evidence of shear banding. 


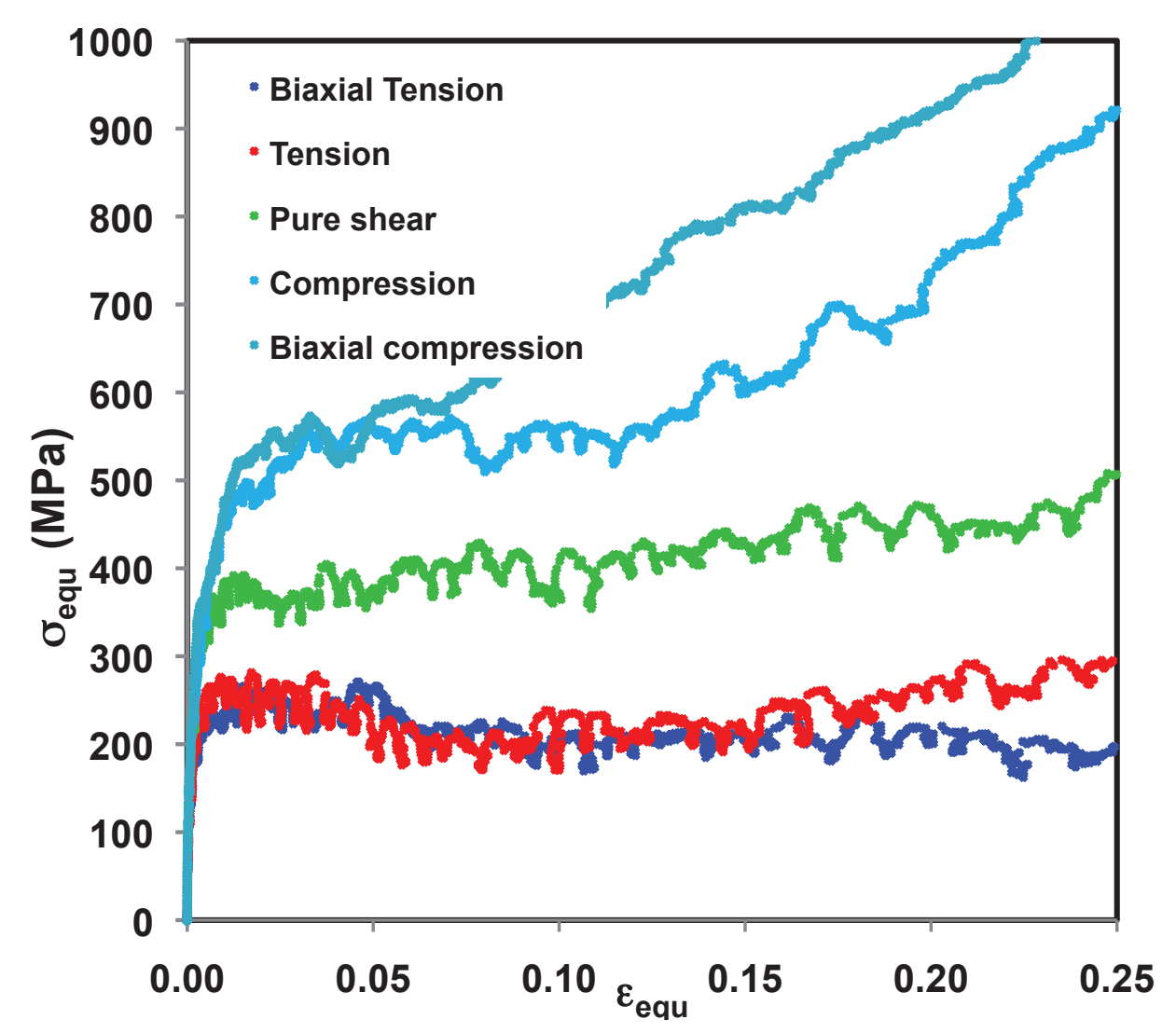

Figure 7. Equivalent stress as a function of equivalent strain for five load cases for system n0. Each data point is the average over one hundred 0.05 fs time steps.

321 The hydrostatic pressure and volumetric dilatation variations in these same five load cases indicate their role in affecting yield and flow behavior. Mean stress is plotted versus $J_{1}$ in Figure 8. In tension and biaxial tension, there is initially a proportional increase in mean stress with $J_{1}$. Upon yield, there is a dramatic rise in $J_{1}$ with a slight decline in mean stress. The system subsequently stiffens up to the point of bond rupture. In pure shear, there is essentially no dilatation (i.e., no increase in $J_{1}$ ) until yield. There is then a gradual increase in mean stress with $J_{1}$ followed by a regime in which the system essentially resists all further dilatation. In both compression cases, $J_{1}$ monotonically decreased at a rate which was similar among the two cases.

The dependencies of energy associated with molecular distortion and non-bond interactions are shown in Figure 9. Bonds generally become the most strained in tension cases. In compression cases, bonds initially become generally less loaded as equivalent strain increases from zero and up to yield. Subsequently, bonds take on additional load at approximately the same rate as the other cases. After yield, van der Waals energy is larger in tension cases than shear and compression. Because the structure has dilated in the tension cases, this rise is due to increase in non-bond attraction. To further explore the interplay among bond and pairwise terms during deformation, the contributions of these to the normal stress terms of the stress tensor were 
compared among the biaxial tension and biaxial compression cases. The results are shown in Figure 10. It was found that bond and pairwise interactions made the largest contribution to the stress tensor. As a result of crosslinking, there are initially positive contributions from bond terms and negative contributions from pairwise terms. When the system is in biaxial extension, the rate of change in the contributions in all directions have similar magnitudes. When the system is placed in compression, however, there is comparatively little change in the contributions in the $x y$ plane while the changes in the $z$ direction are large and nonlinear. As this occurs, the material is extruded in the $z$ direction.

In tension cases, torsional energy increases gradually and becomes an order of magnitude smaller than the compression cases. It therefore appears positive mean stress has the effect of dilating the structure to a threshold level at which some barriers to chain reconformation are removed and plastic flow proceeds. Once initiated, it appears there are then numerous conformations available to the chains at similar or lower energy levels. Conversely, negative mean stress (and the nearly proportional contraction) appears to allow rearrangements with the addition of increasing amounts of work for each strain increment. These effects of mean stress are consistent with experiments. Sternstein and Ongchin inferred that positive mean stress enhances conformational mobility [11]. Knauss and Emri [74] show that the effect of increasing hydrostatic pressure on glassy polymers is to delay yield and suppress post-yield softening.

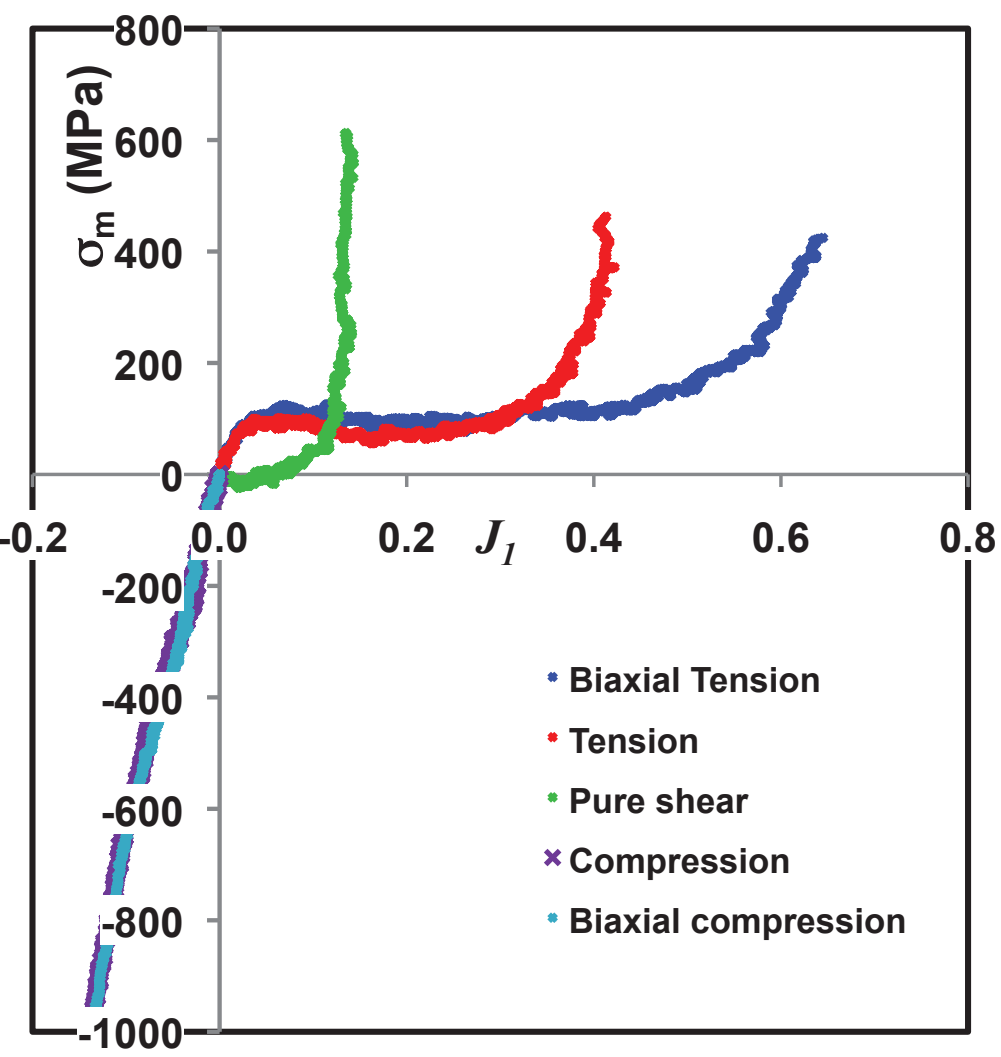

Figure 8. Mean stress as a function of the first invariant of the strain tensor for system $\mathbf{n 0}$. 


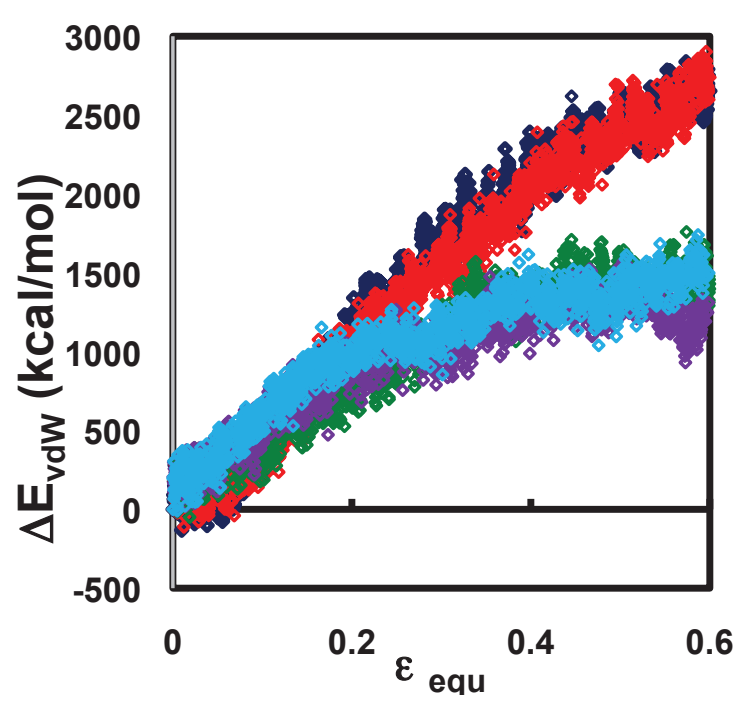

362

a)
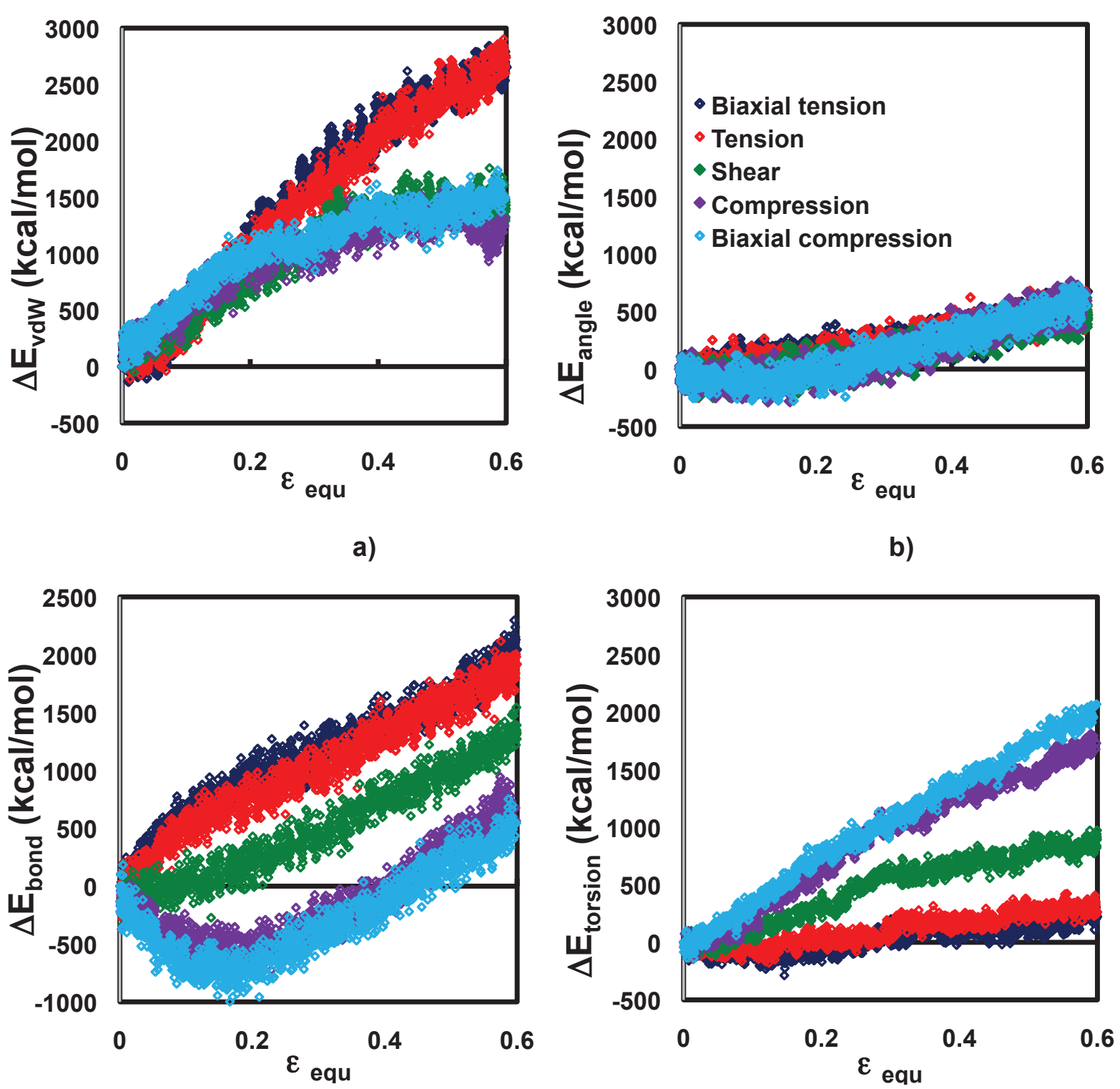

d)

Figure 9. The dependencies of van der Waals (a), angle (b), bond (c), and torsional (d) system energies on equivalent strain for five load cases for system no. Columbic interaction energies are not shown because the magnitude of the variations was distinctly smaller. 
368

369

370

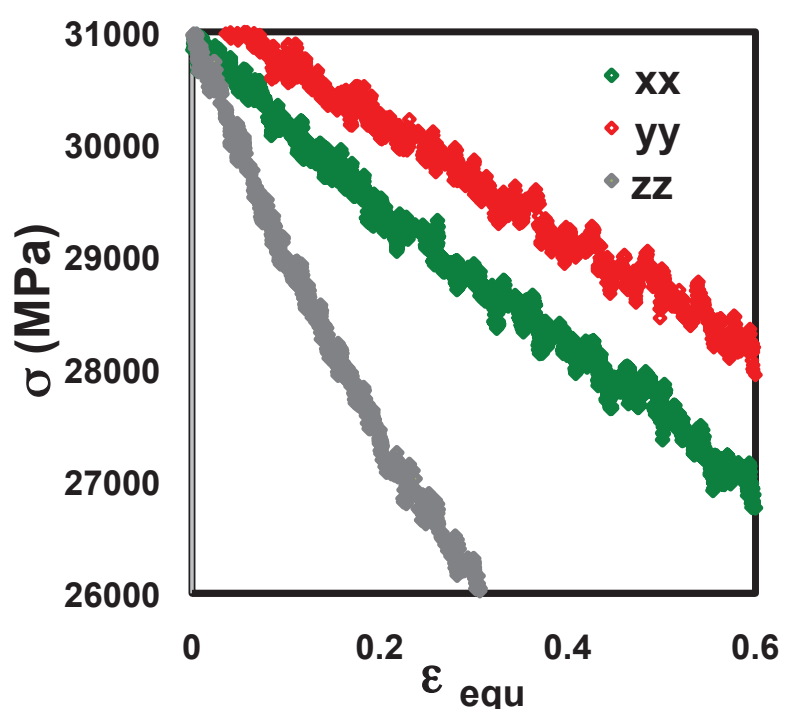

a)

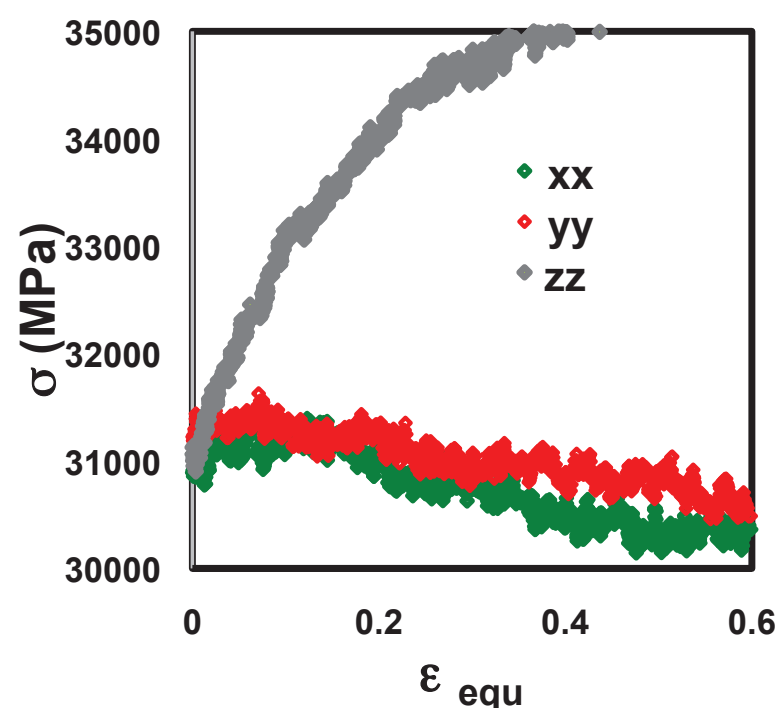

c)

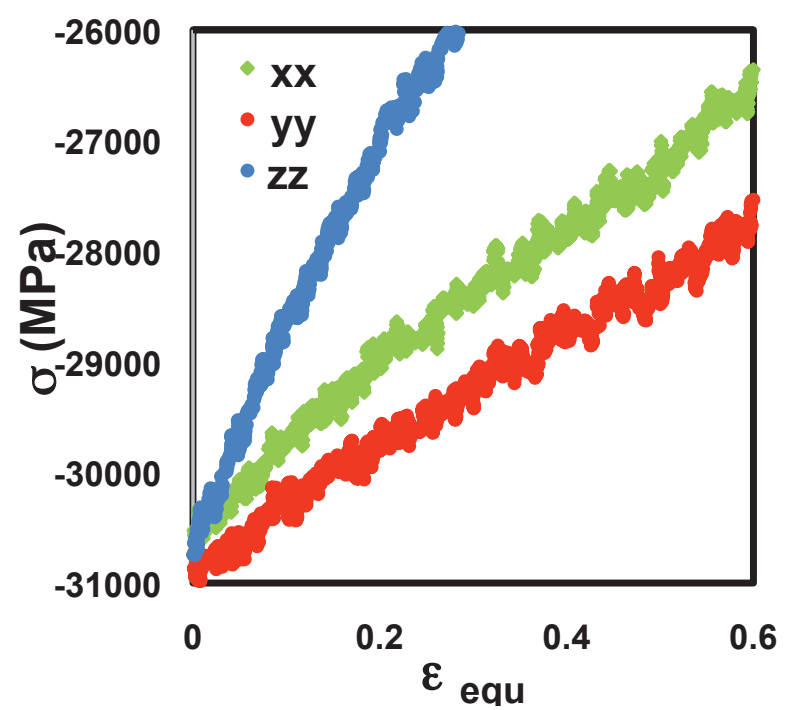

b)

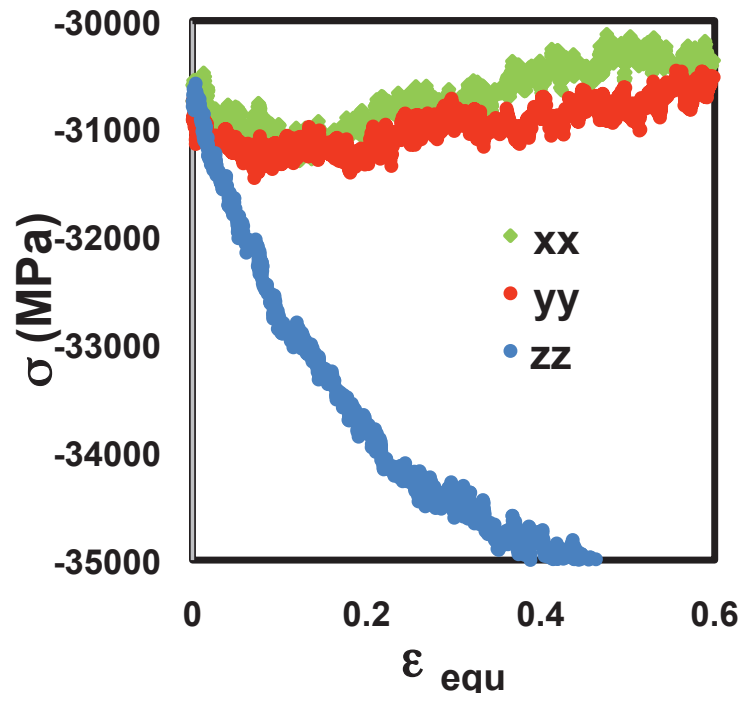

d)

Figure 10. Net contributions of bond terms (subfigures a and c) and pair-wise terms (subfigures $b$ and d). Subfigures a and $b$ are for biaxial tension. Subfigures $c$ and $d$ are for biaxial compression.

\section{Elastic Properties}

In system n0, the bulk moduli in the limit of zero strain were 5.6 and $5.8 \mathrm{GPa}$ and for biaxial tension and biaxial compression, respectively. These compare well with a measured value of $5.4 \mathrm{GPa}$ measured for a DGEBA-TETA system [18]. These predictions also show distinctly better agreement compared to our previous predictions [48] for the same systems using the COMPASS force field. Effective modulus was treated as the initial slope of the equivalent stress-equivalent strain curve. The results show modulus was generally lowest in biaxial tension and generally increased with the largest value in biaxial compression (as shown in a 
polar plot for each system in Figure 11). Modulus also increased nonlinearly with crosslink density. While there is a three-fold difference in crosslink density among systems $\mathrm{n} 0$ and $\mathrm{n} 3$, moduli differed by a far smaller proportion. Further, crosslink density in system n5 was $68 \%$ that of system $\mathrm{n} 3$ whereas its modulus was a half to a third that of system $\mathrm{n} 3$. This strong nonlinear dependence upon cross-link density is consistent with experiments of Charlesby and Hancock [75] who found (consistent with the theory for amorphous cross-linked polymers) that modulus varied inversely with molecular weight between crosslinks.

Elastic modulus and Poisson's ratio were also inferred from the results. Assuming the material 397

Figure 11. Polar plot of effective elastic modulus for systems n0, n3, and n5. The angular position of each point corresponds to the angle $\theta$ in Figure 1 associated with the ratio of the in-plane stresses for the respective case.

to be linearly elastic at small strain, stress and strain in plane stress (in the $x y$ plane) are related by

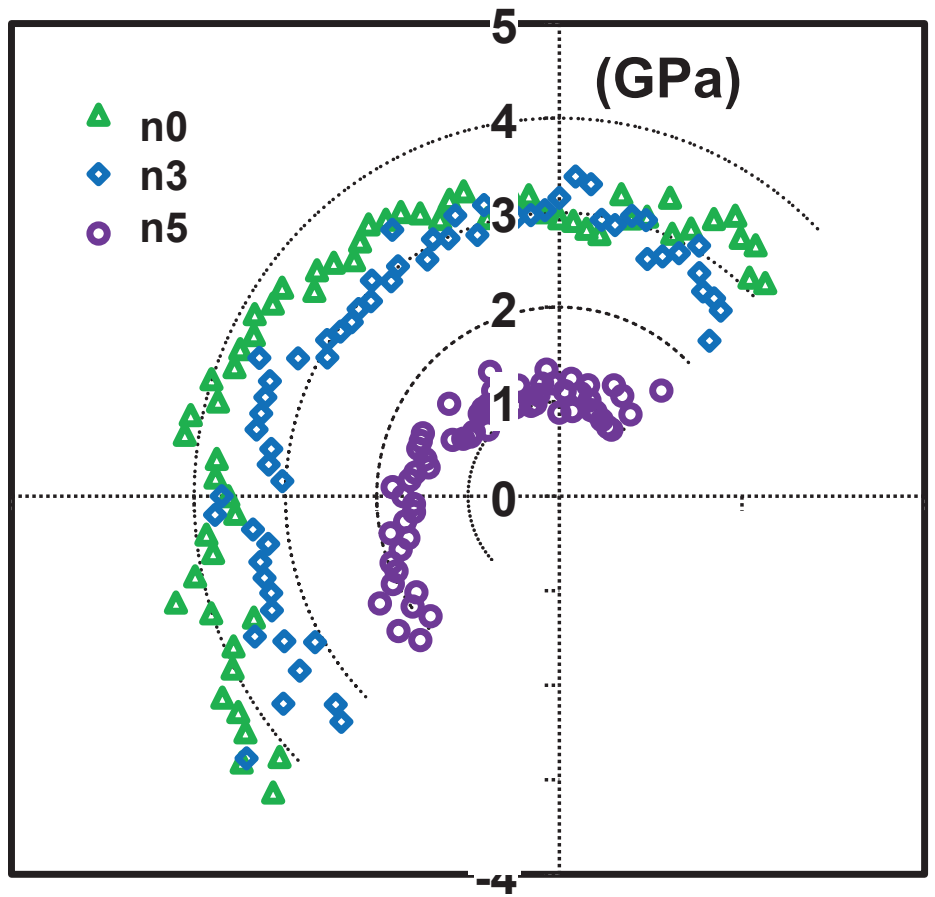

399

$$
\varepsilon_{x}=\frac{1}{E}\left[\sigma_{x}-v \sigma_{y}\right]
$$

400

$$
\varepsilon_{y}=\frac{1}{E}\left[\sigma_{y}-v \sigma_{x_{-}}^{-}\right.
$$

which can be rearranged as

401

$$
E=\frac{\sigma_{x}^{2}-\sigma_{y}^{2}}{\sigma_{x} \varepsilon_{x}-\sigma_{y} \varepsilon_{y}}
$$




$$
v=\frac{\frac{\varepsilon_{x}}{\varepsilon_{y}}-\frac{\sigma_{x}}{\sigma_{y}}}{\frac{\varepsilon_{x}}{\varepsilon_{y}} \frac{\sigma_{x}}{\sigma_{y}}-1}
$$

Most of the inferred elastic moduli ranged from 1.5 to $3.0 \mathrm{GPa}$ with the largest in the range between pure shear and compression (as shown in Figure 12). Littell, et al. [76] tested an EPON 862/Epikure W system (i.e., DGEBF resin 165-173 e.w. and non-MDA aromatic curing agent 4346 a.h.e.w.) and found moduli in compression and tension of 2.96 and $2.89 \mathrm{GPa}$, respectively. While Littell's results showed weak dependence of modulus on load type, Kaelble [77] found such dependence varied with the crosslinking molecule used in the formulation. His measured values ranged from 1.31 to $3.50 \mathrm{GPa}$. He found DGEBA (200 e.w.) crosslinked with the comparatively flexible DETA resulted in a system which was stiffer in tension than compression while the formulation crosslinked with the shorter metaphenylene diamine led to the opposite behavior. The behavior found here is consistent with Kaelble's findings in that the crosslinking molecule used is also comparatively short and stiff (being composed of two phenyl groups bonded by one carbon atom).

416 The inferred Poisson's ratio ranged primarily from 0.25 to 0.50 (as depicted in Figure 13). 417 Sottos, et al. [78] found values ranging from 0.40 to 0.49 for an EPON 828 (DGEBA 185-192 418 e.w.) system fully-reacted with an aliphatic curing agent. Littell, et al. [76] found values ranging from 0.35 to 0.43 at room temperature for EPON 862 (DGEBA 165-173 e.w.) cured with a modified polyamidoamine agent. Smith, et al. [18] also found the values ranged from 0.25 to 4210.50 for an amine-cured DGEBA and that the value was highest in compression and decreases 422 with the addition of shearing.

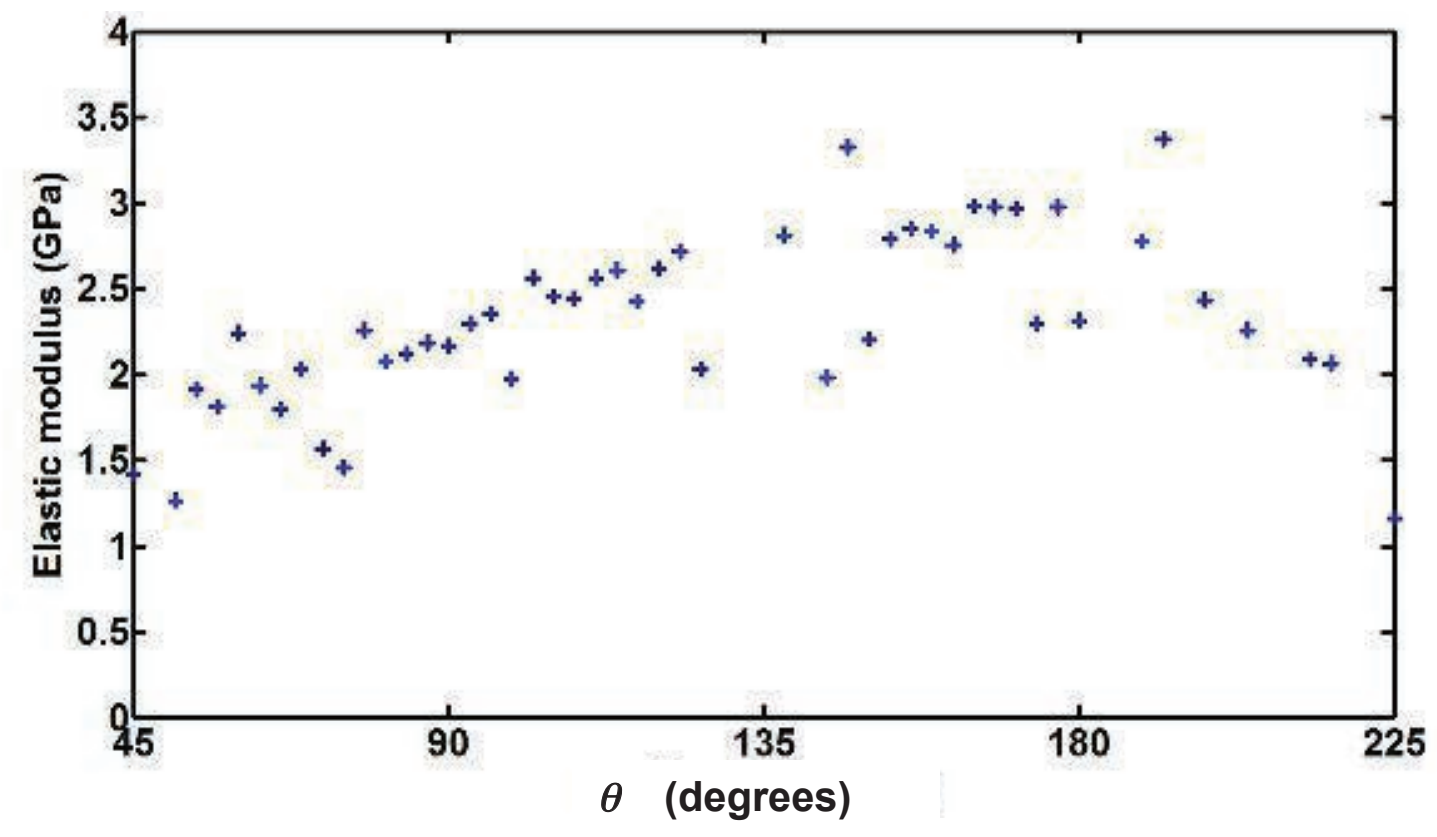


Figure 12. Small-strain elastic modulus over the range of load cases for system n0. Biaxial tension and biaxial compression are on the extreme left and right, respectively.

426

427

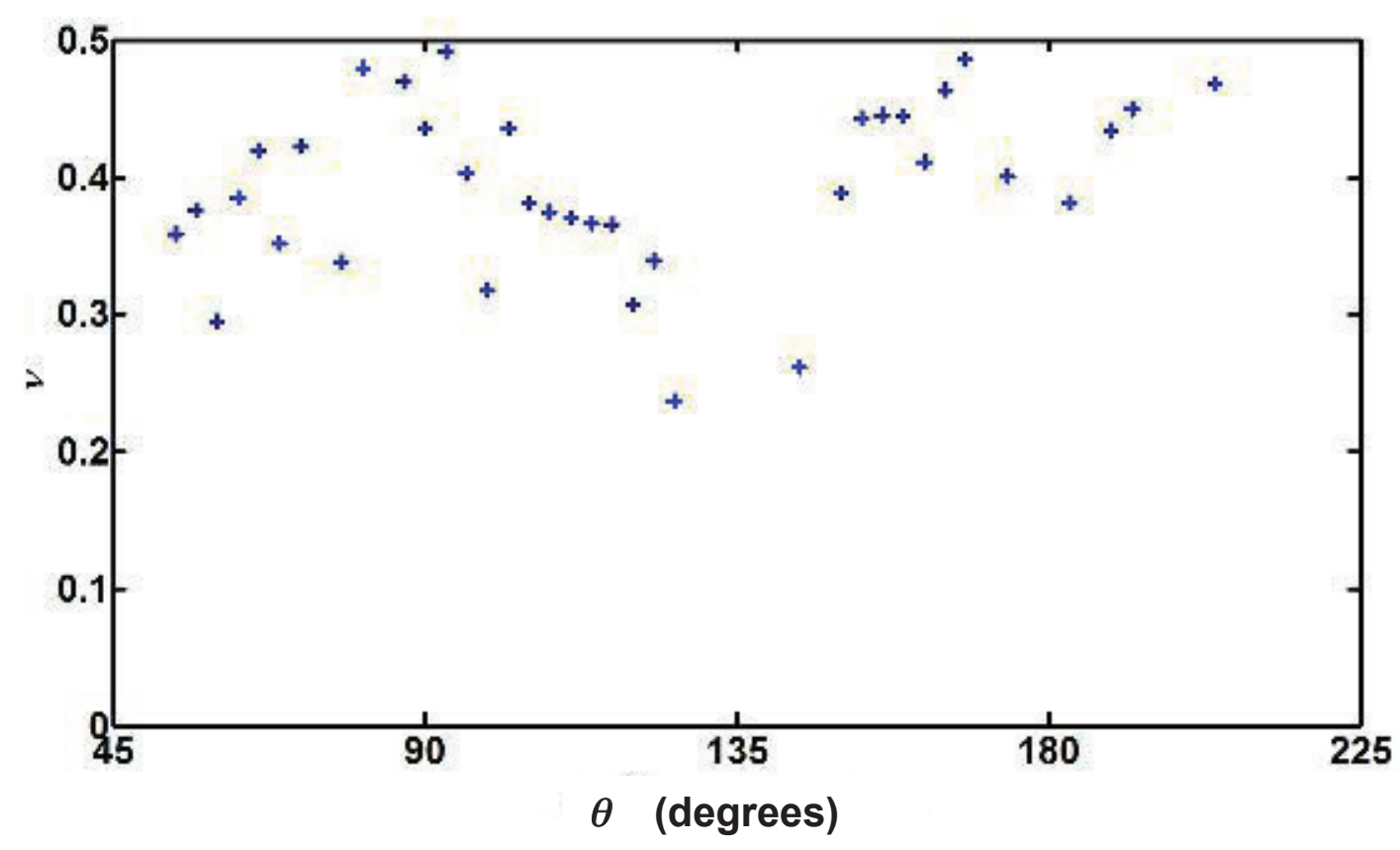

Figure 13. Inferred Poisson's ratio over the range of load cases for system n0. Biaxial tension and biaxial compression are on the extreme left and right, respectively.

\section{Yield}

Yield points were identified from fits to the respective stress-strain curves. For responses which included strain softening, yield was defined as the first point of zero slope at which the second derivative was positive (i.e., lower yield). When responses did not include strain softening, the first inflection point was treated as the yield. These features could be easily identified in all simulation results. Lower yield was deemed more appropriate than upper because the results of Girard-Reydet, et al. [79] indicate that strain is not recovered in the former while it is in the latter.

Consistent with the experiments of others, dependence of yield on pressure was found. When compressive, the general effect of hydrostatic stress is to contract the system and therefore reduce the available space for chain segment mobility. When yield strength is plotted as a function of $I_{1}$ (as in Figure 14), a linear relation is found for a wide range where strength decreases with increasing hydrostatic stress. Pressure-dependent yield has been found for PC and PVC [14], PMMA [11], and cured DGEBA [9, 8, 12]. The sensitivity of yield strength to $I_{1}$ was $0.53 \mathrm{MPa} / \mathrm{MPa}$. This level is greater than experimentally-measured values (ca. 0.1 for PMMA [11], 0.164 for DGEBA/EDA/MEDA/DMEDA [8], 0.175 for DGEBA/diisocyanate [9], and 0.46 for DGEBA/DETA [12]).

The predicted yield values are larger than found from the compression tests of DGEBA cured with various agents by Cook and Mayr [80]. However, these experiments were performed at lower strain rate and they also found distinct hardening with strain rate. Also, the yield values 
predicted here show distinctly better agreement than our previous predictions using the COMPASS force field [48].

451 Another frequently-observed phenomenon, a different dependence on invariants in the first 452 quadrant of the $\sigma_{1}$ versus $\sigma_{2}$ plot compared with the second and third quadrants, is also present. 453 In Figure 14, yield values from the first quadrant are shown as filled symbols. They tend to 454 cluster within a narrower range of $I_{1}$ than those from the other quadrants and not display a 455 discernible linear dependence.

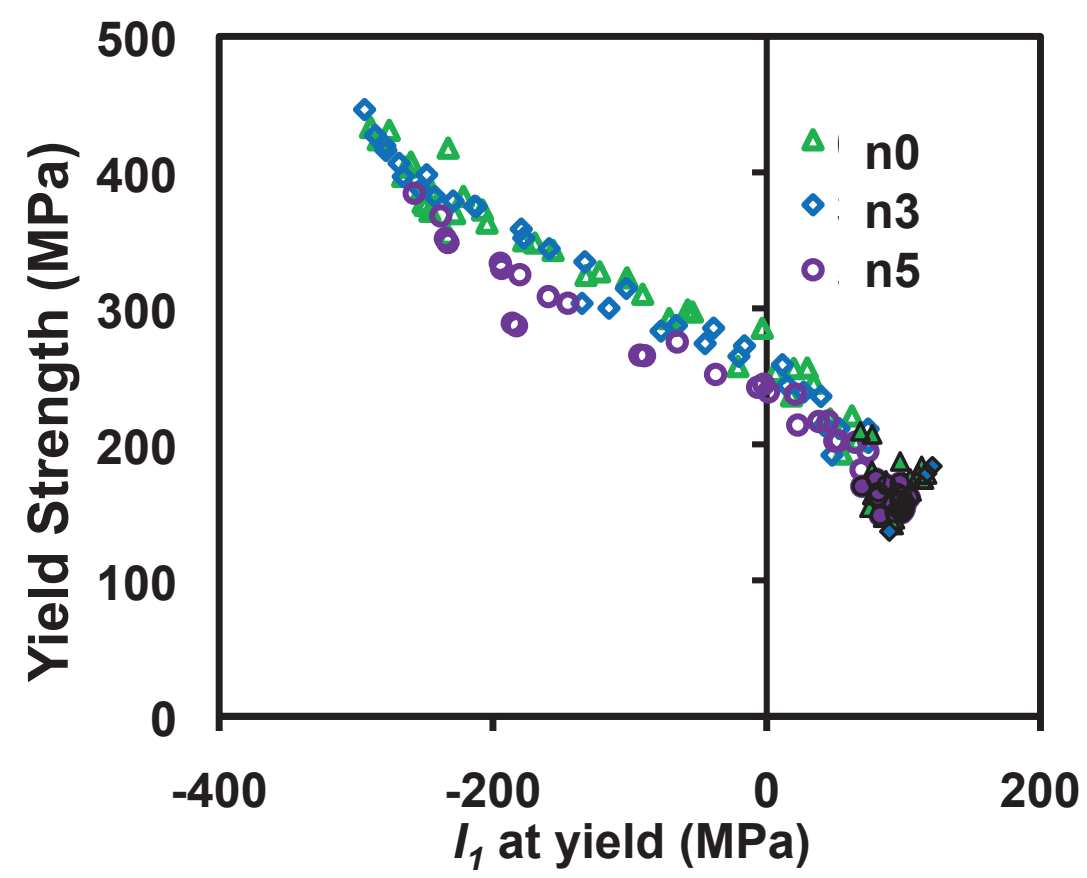

Figure 14. Equivalent stress at yield as a function of the first stress invariant. Filled symbols with dark borders represent first quadrant results.

To further illustrate, results were compared with several commonly-used yield criteria to more completely investigate invariant dependence. The von Mises criterion is given by

$$
\sqrt{\frac{1}{6}\left[\left(\sigma_{1}-\sigma_{2}\right)^{2}+\left(\sigma_{2}-\sigma_{3}\right)^{2}+\left(\sigma_{3}-\sigma_{1}\right)^{2}\right]}=\sqrt{\frac{2}{3}\left(I_{1}^{2}-3 I_{2}\right)}=\frac{1}{2} s_{i j} s_{i j}=K^{2}
$$
on the yield strength in uniaxial tension $S_{Y t}$ by

$$
K=\frac{S_{Y t}}{\sqrt{3}}
$$




$$
\sqrt{\frac{1}{6}\left[\left(\sigma_{1}-\sigma_{2}\right)^{2}+\left(\sigma_{2}-\sigma_{3}\right)^{2}+\left(\sigma_{3}-\sigma_{1}\right)^{2}\right]}=\sqrt{\frac{1}{3}\left(I_{1}^{2}-3 I_{2}\right)}=A+B I_{1}
$$

where

$$
A=\frac{2}{\sqrt{3}}\left(\frac{S_{Y c} S_{Y t}}{S_{Y c}+S_{Y t}}\right) \quad, \quad B=\frac{1}{\sqrt{3}}\left(\frac{S_{Y t}-S_{Y c}}{S_{Y c}+S_{Y t}}\right)
$$

472

473

474

475

476

477

478

and the subscript $c$ denotes compression.

The modified Tresca criterion includes pressure-dependent threshold for the maximum shear stress. That is

$$
\left|\sigma_{M A X}-\sigma_{M I N}\right|=2\left(\frac{S_{Y c} S_{Y t}}{S_{Y c}+S_{Y t}}-\frac{1}{2} \frac{S_{Y c}-S_{Y t}}{S_{Y c}+S_{Y t}} I_{1}\right)
$$

A normal-stress-yielding criterion for plane stress was derived by Sternstein and Onchin [11] to match some of their results

$$
\left|\sigma_{1}-\sigma_{2}\right|=A+\frac{B}{I_{1}}
$$

This criterion was based on assertions that $I_{1}$ was a factor determining molecular mobility and $\left|\sigma_{1}-\sigma_{2}\right|$ drives normal yielding. This criterion appeared to hold under conditions in which this threshold would lie within the von Mises envelope.

Based on some of the first-quadrant results of Asp, et al. [12], another test criterion is setting the first invariant equal to the tensile yield strength.

$$
I_{1}=S_{Y t}
$$

Fits to the data according to these criteria for system n0 are shown in Figure 15. The DruckerPrager criterion overpredicts strength in the first quadrant and provides a very good fit in the second and third. In the third quadrant, both Drucker-Prager and modified Tresca criteria bear resemblance to the trend in the data. There is enough variability in the results that it is unclear which criterion is a better fit; especially in conditions approaching biaxial compression. The modified Tresca and Sternstein-Ongchin criteria provide the best fits in the first quadrant. While both provide for weakening with increasing $I_{1}$, they also overestimate strength for conditions approaching biaxial tension. Data in this vicinity tend to form a line parallel to the constant- $I_{1}$ line. As shown in Figure 16, the other systems showed a similar general behavior. Also, strength increased with increasing crosslink density.

While some polymers have displayed an $I_{1}$-dependent dichotomy in their responses to biaxial stress, others do not. For thermosets, it appears the type of first-quadrant response depends on the cross-linking molecule flexibility. Asp, et al. [12] tested DGEBA cured with diethylene 
triamine (DETA) as well as DGEBA with poly(oxy propylene-amine) (APTA, Jeffamine T-403). While both are aliphatic triamines, the latter has a longer backbone contour length (i.e., DETA has six backbone bonds and APTA has approximately 24). The results showed DGEBA/DETA yield strength could be fit to a constant $I_{1}$ criterion in the first quadrant whereas the DGEBA/APTA formulation could be better fit to von Mises or Drucker-Prager criteria. In the results obtained here, strength was consistently between Drucker-Prager and constant- $I_{1}$ fits in the first quadrant. Similar to the DGEBA/DETA formulation, the crosslinking molecule used here, MDA, is also comparatively short.

510

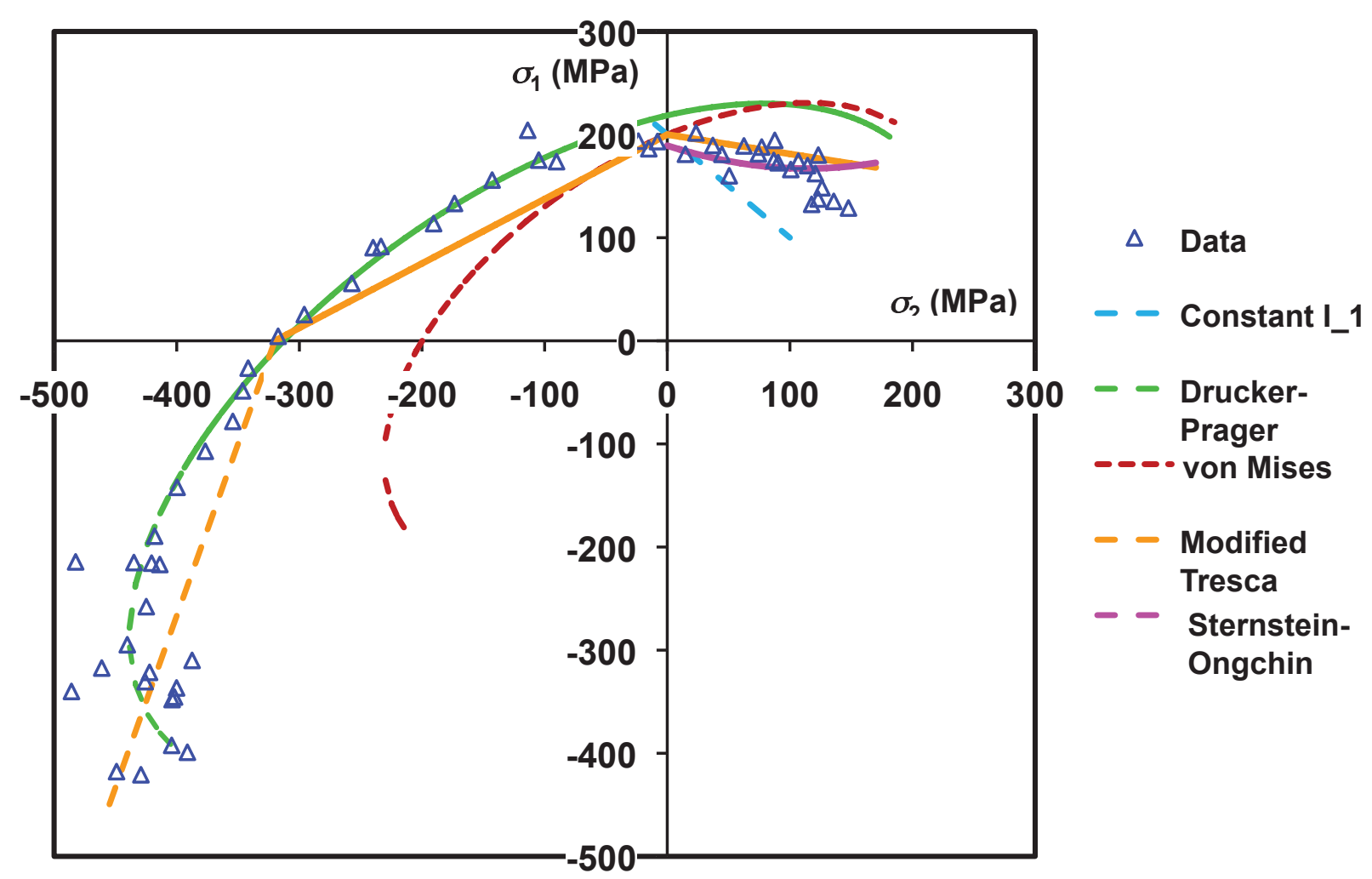

Figure 15. Comparison among several yield models fit to the system n0 results. 


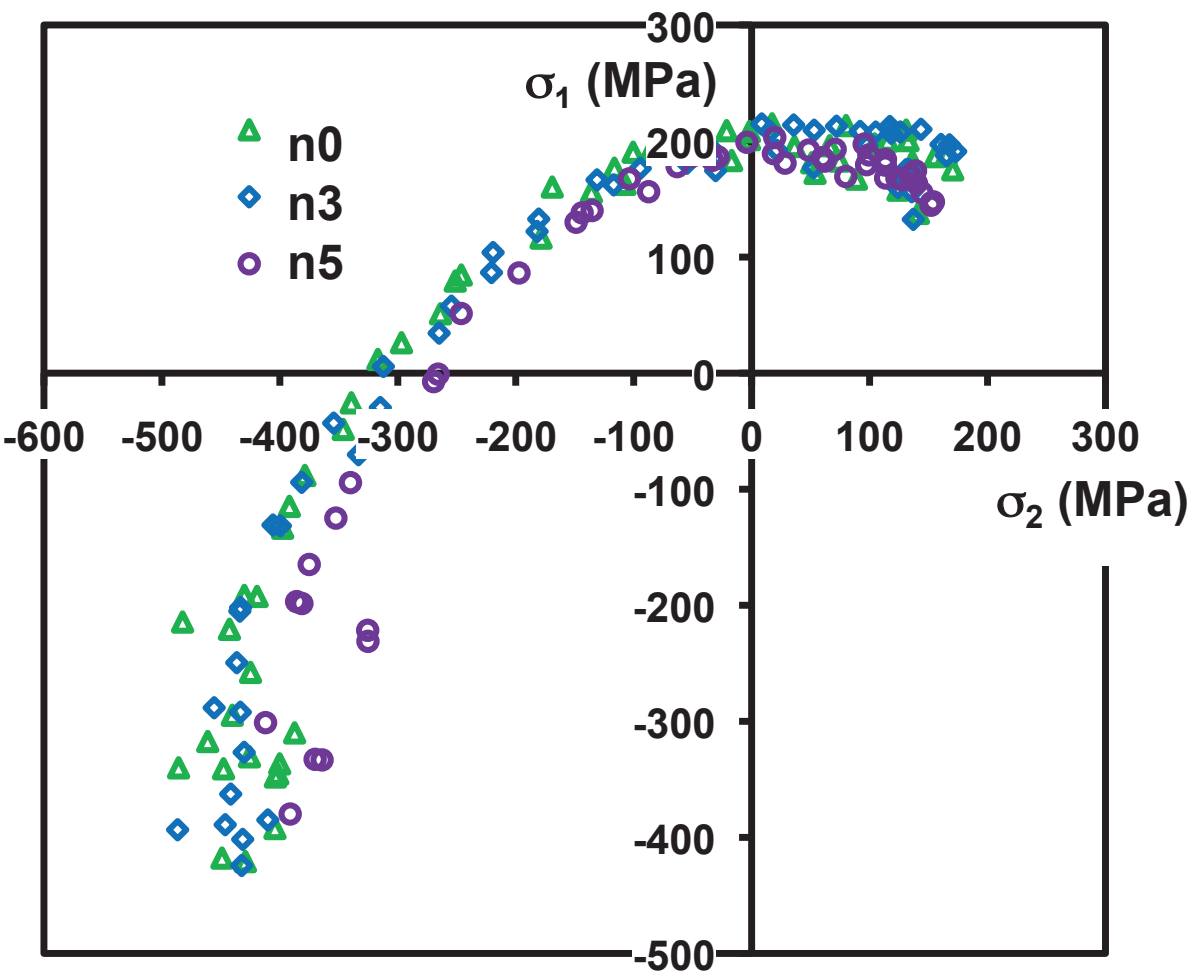

Figure 16. Plot of principal stresses at yield for systems n0, n3, and n5.

It has been previously found that when this dichotomy in the yield dependence is present, it is associated with differing macroscopic features (e.g., voids or shear bands) [10, 11]. While the cell length scales here are too small to directly depict these, the results here display features which may be precursors. Because mean stress is one of the factors which affects system dilatation, $J_{1}$ at yield is plotted as a function of $I_{1}$ in Figure 17. Dilatation generally increases with mean stress. System no (with largest crosslink density) has the largest $J_{1}$ value for all conditions. The weaker dependence of $J_{1}$ on $I_{1}$ in compression is associated with the increased barriers to torsional rearrangement (as indicated in Figure 9). Further, the more open structure at low crosslink density (system n5) appears to enable better packing as the system is deformed. For system n5, contraction also occurred in some instances where $I_{1}$ was positive. It therefore appears that this contraction was at least partly enabled by the presence of shearing. In all instances of first-quadrant yield, dilatation was positive. To highlight this, first-quadrant points are shown as closed symbols in Figure 17. There is a dramatic increase in the amount of dilation between uniaxial tension (the lowest solid symbols) and biaxial tension (the highest). Reminiscent of the results of Asp, et al. [12], there is a narrow range of $I_{1}$ at which yield occurred.

While the dilatation at yield in the first quadrant appears to be generally uniform across each system, there is evidence that post-yield flow would readily lead to void growth. The free volume in system n0 was investigated. Free volume was defined here as the portion of the cell not 
enclosed by the van der Waals diameter of any atom. The diameter of the largest sphere which could be placed entirely within the free volume was monitored as deformation proceeded. The result for several cases is shown in Figure 18. Most notable is the distinct rise in sphere radius in first-quadrant cases. While these occurred at equivalent strain much greater than the value at yield, it should also be recalled that this system was essentially perfectly plastic in tension and biaxial tension. These results indicate that, if the stress on the system were to be held at the yield value in these two cases, plastic flow would rapidly progress and distinct nano-scale porosity would form (whereas it would not form in the other cases). Such voids may be precursors to a larger-scale rupture event. Indeed, actual thermoset systems have been shown to be less stable (i.e., have a stronger propensity to fracture) in first-quadrant loading. Kody and Lesser [8] found brittle behavior in biaxial tension and simple tension and ductile behavior in uniaxial compression.

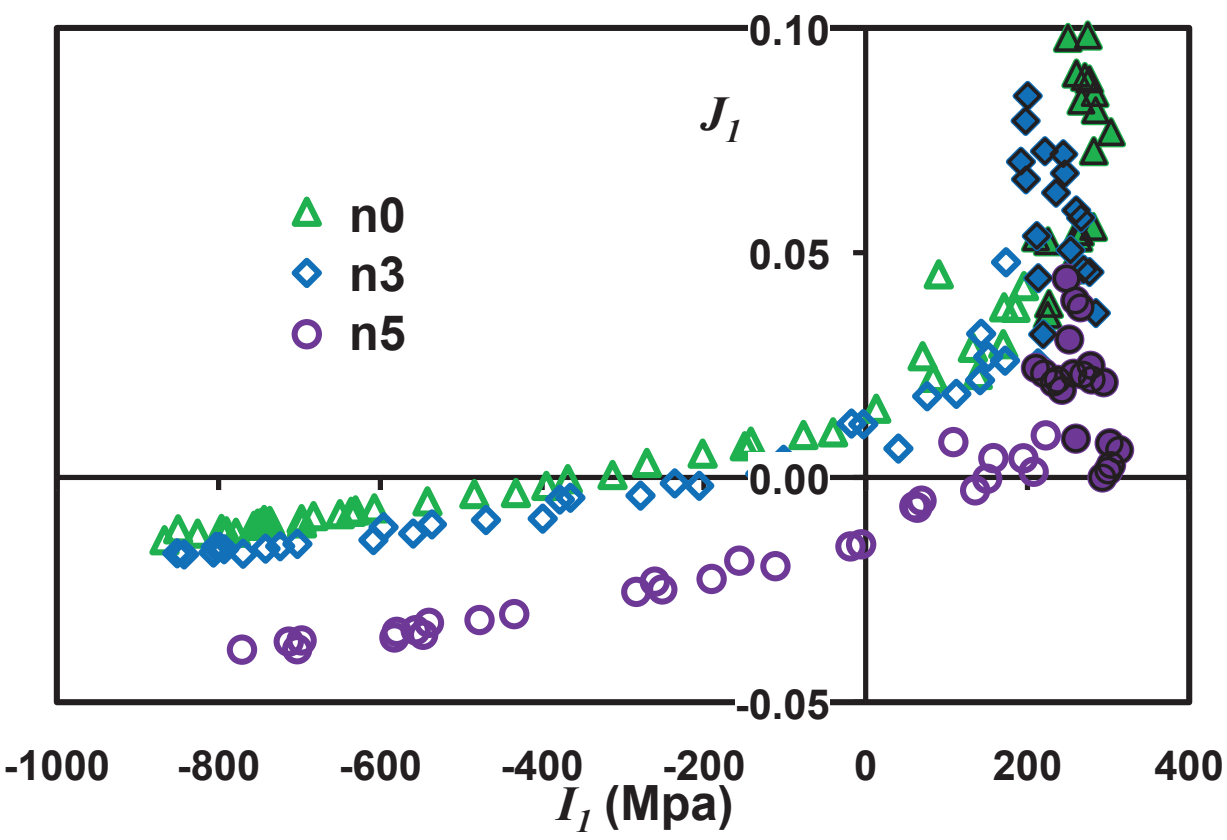




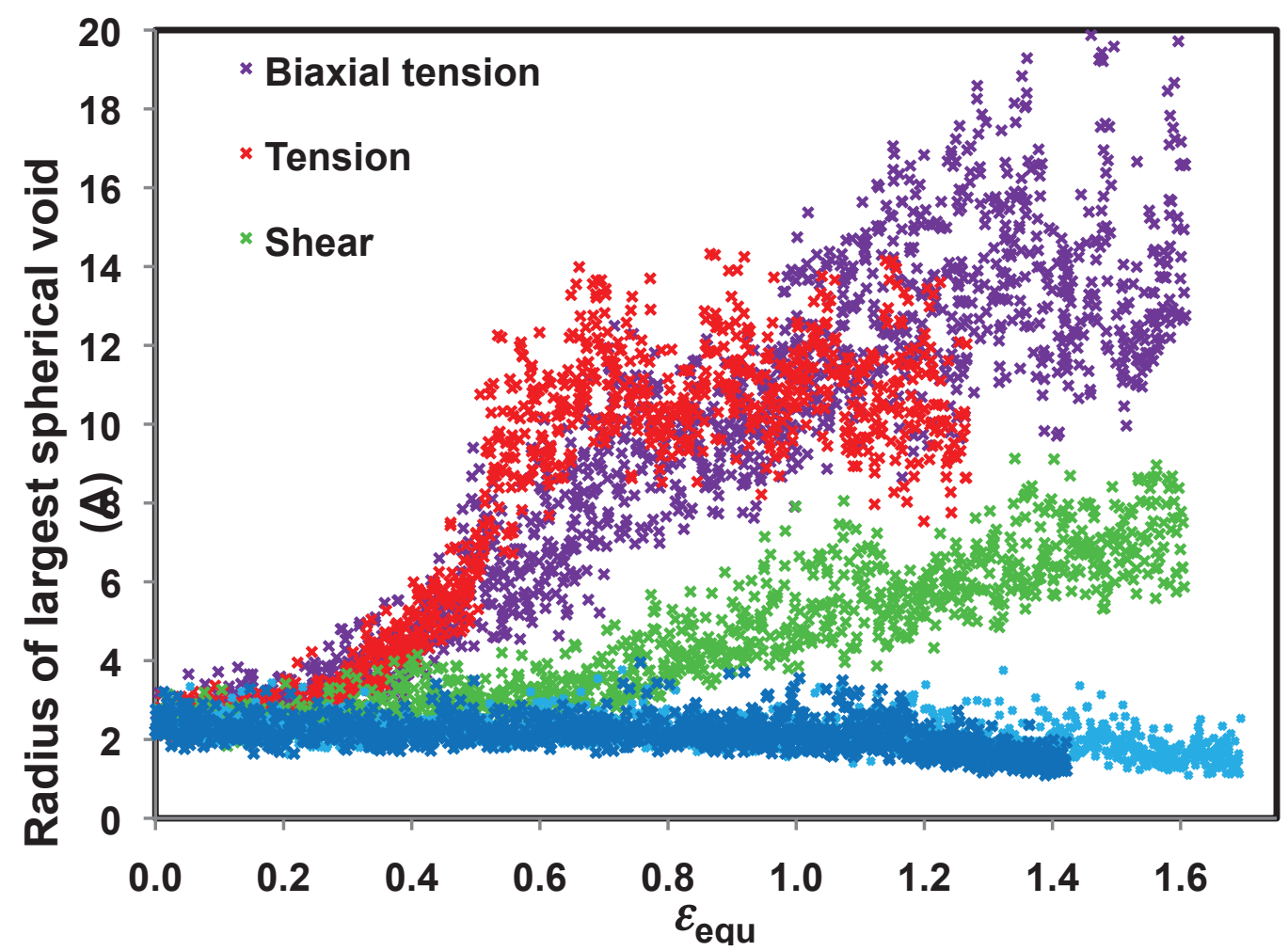

553

554

555

Figure 18. Radius of the largest sphere of free volume found in system $\mathrm{n} 0$ as a function of equivalent strain.

\section{Rupture}

Rupture was here defined as the system state at the first instance of a backbone bond energy reaching or first exceeding its corresponding critical threshold value. These were collected for all systems and numerous load cases. The envelopes differed distinctly from those for yield. As shown in Figure 19, equivalent stress at rupture rises dramatically between biaxial tension and pure tension, rises more gradually between tension and pure compression, and continues to increase with the largest value apparently in biaxial compression. Because each outcome was dependent on the loading of a small fraction of atoms, dependence on crosslink density is difficult to discern.

Ductility was lowest in pure tension and biaxial compression. As inferred from Figure 20, equivalent strain at rupture varied little in the first and second quadrants with the exception of pure tension. It appears the superposition of a small stress level orthogonal to the tensile direction led to sufficient shear stress additional uncoiling and interchain slip. In the third quadrant, ductility generally decreased from compression to biaxial compression. Ductility generally increased with decreasing cross-link density. Between systems $\mathrm{n} 0$ and $\mathrm{n} 3$, crosslink density decreased by nearly three-fold from $1.4 \times 10^{-3}$ to $0.48 \times 10^{-3} A^{-3}$. When comparing these two systems, ductility distinctly increased for all load cases. The crosslink density among systems $\mathrm{n} 3$ and $\mathrm{n} 5$ differed by too small an amount $\left(0.33 \times 10^{-3} A^{-3}\right)$ to infer a trend. There have been experiments in which large ductility has been observed in epoxy. For example, Glad and Kramer [60] recorded stretch values in tension as high as 1.6 for the same systems as 
576 those tested here. Cook and Mayr [80] observed strain to rupture in compression was an order 577 of magnitude greater than the yield strain.

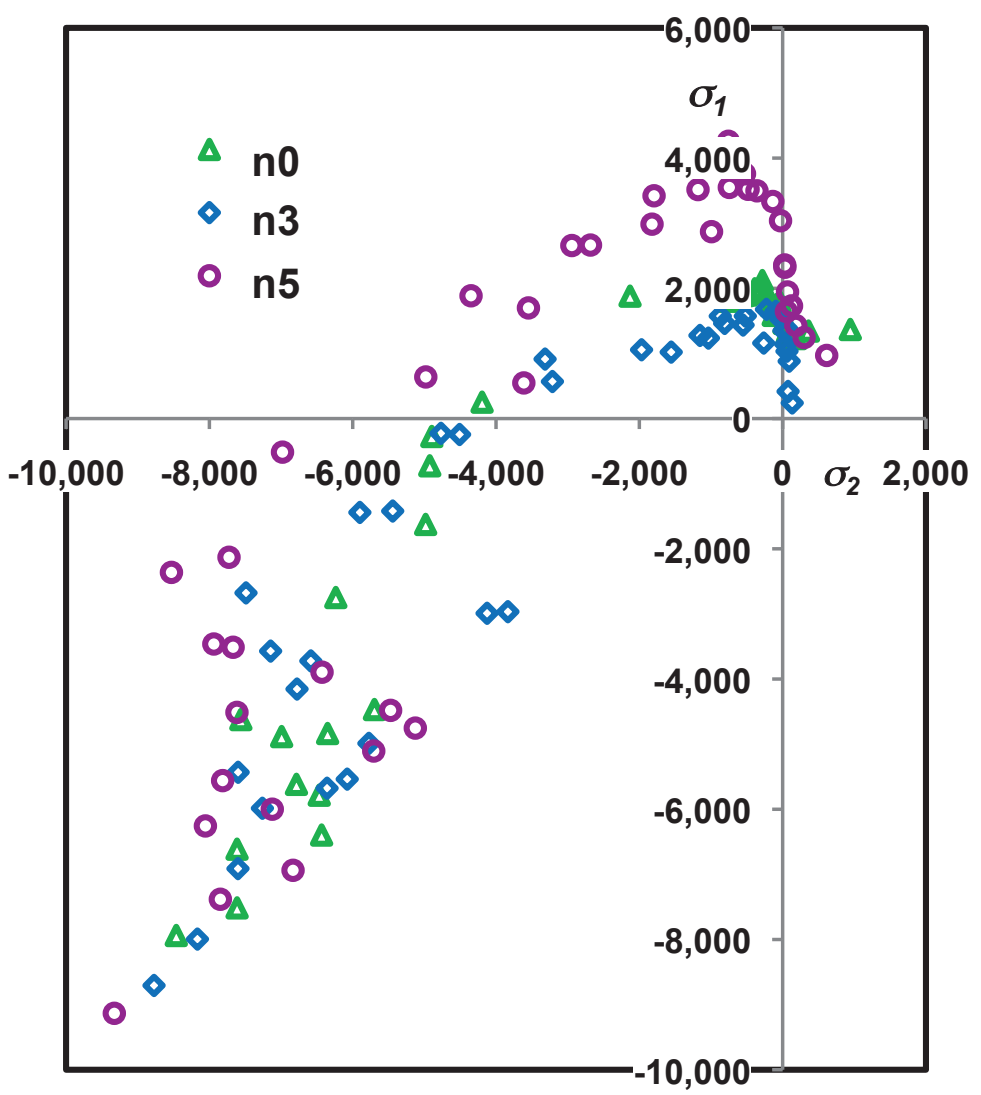

Figure 19. $\sigma_{1} \sigma_{2}$ plot of stress at rupture. Values are MPa.

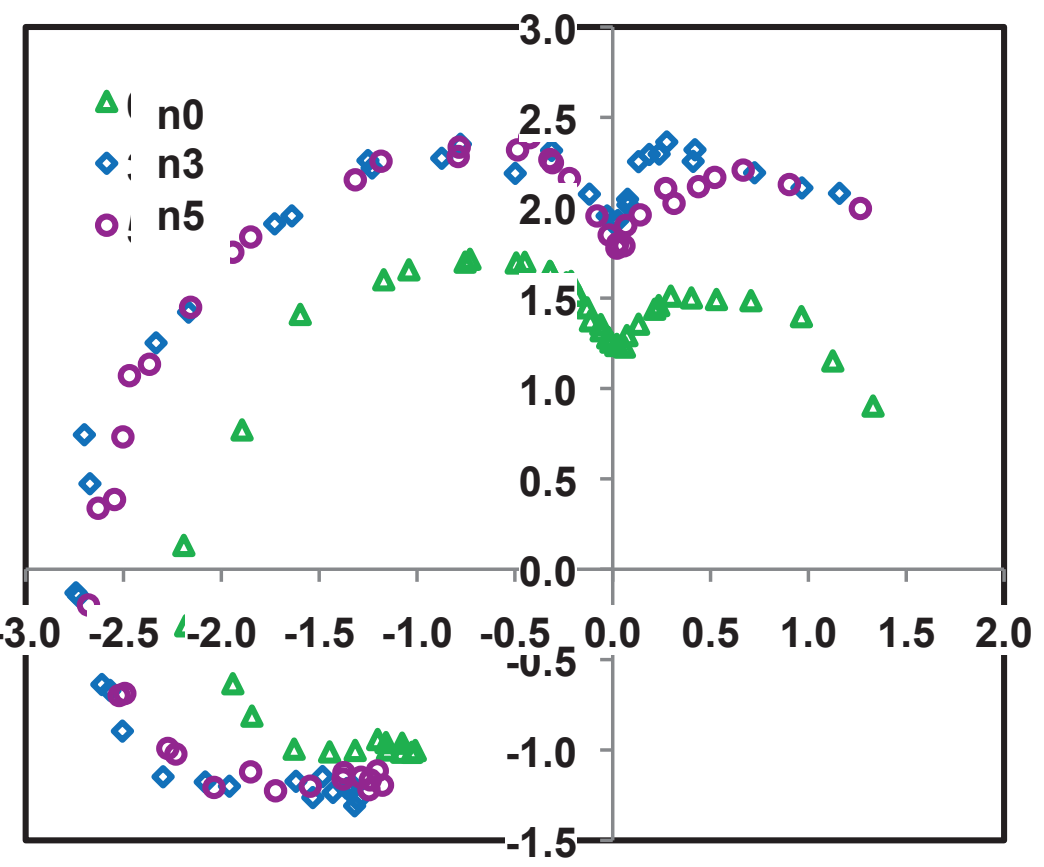


Figure 20. Equivalent strain at rupture in the form of a polar plot. Polar angle corresponds to principal stress ratio.

Several workers $[8,10,11]$ observed distinct differences in the nature of yield and rupture between first quadrant loading and other loading cases. As shown in Figure 21, the level of the first strain invariant at rupture here showed a similar dichotomy among the quadrants. The experiments of [10] on several thermosets inferred first-quadrant $J_{1}$ values at rupture ranging from ca. -0.005 to 0.015 . Increasing cross-link density tended to decrease the amount of dilation in the first quadrant.

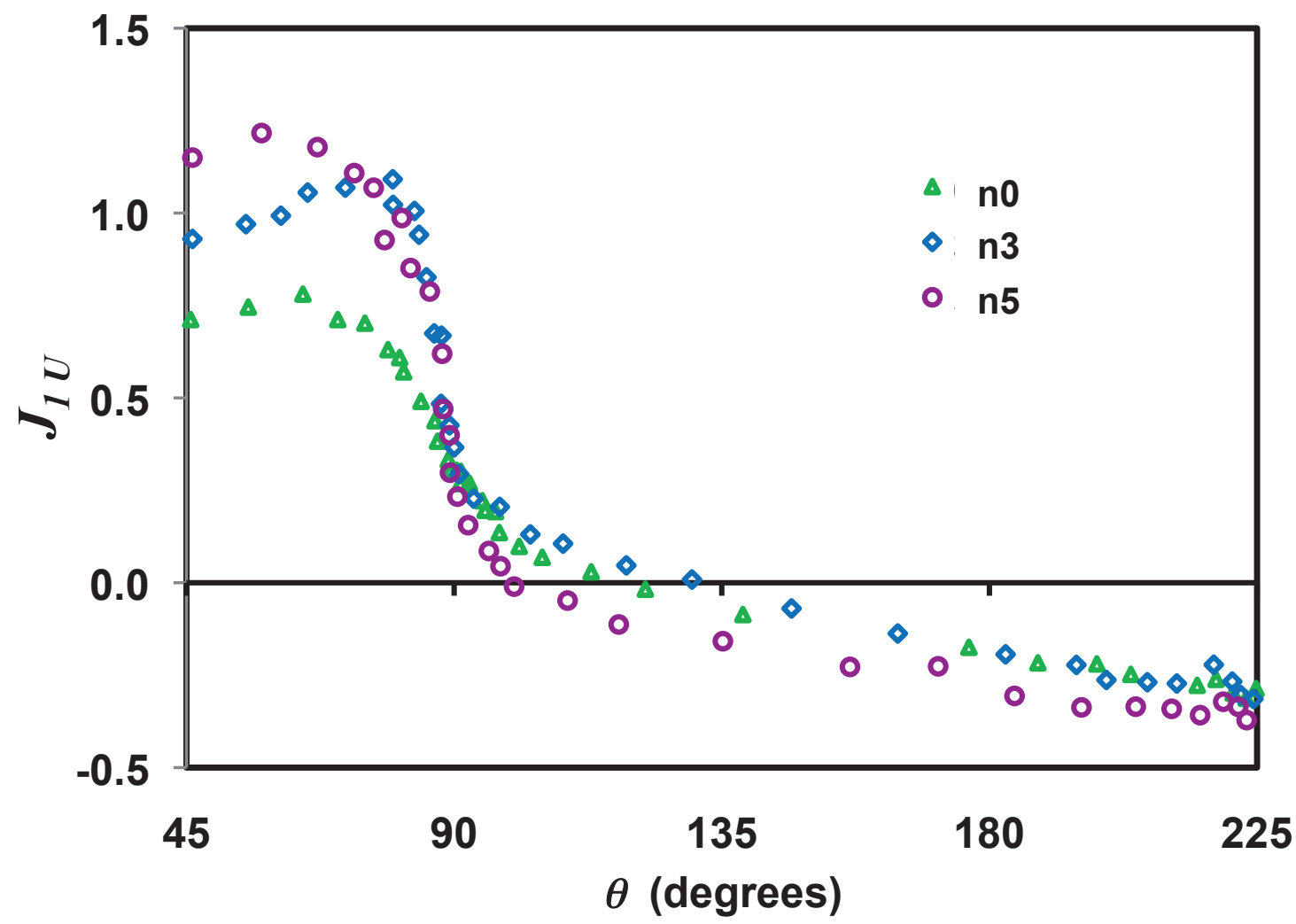

Figure 21. Value of strain tensor first invariant as a function of in-plane principal stress ratio.

The bond type which was first to reach its rupture threshold provides insight into the nature of molecular loading in each load case as well as the possible fracture nucleation sites. Figure 22 shows ruptured bond type according to case for all systems. The most common bond type was between phenyl carbon and aliphatic carbon atoms. In cases for which there was a comparatively large tensile stress in one direction, however, either the carbon-nitrogen bond at the crosslink site or, for system n5, the backbone ether bond was the rupture site. When taken together with the result shown in Figure 20, it appears the lower ductility in cases approximating pure tension is related to the bond type which ruptures. Where there have been numerous experiments to determine the radicals formed from bond scission during tensile deformation of thermoplastics [81], there are no known results from experiments on thermosets against which to compare the results here. 
605

606

607

608

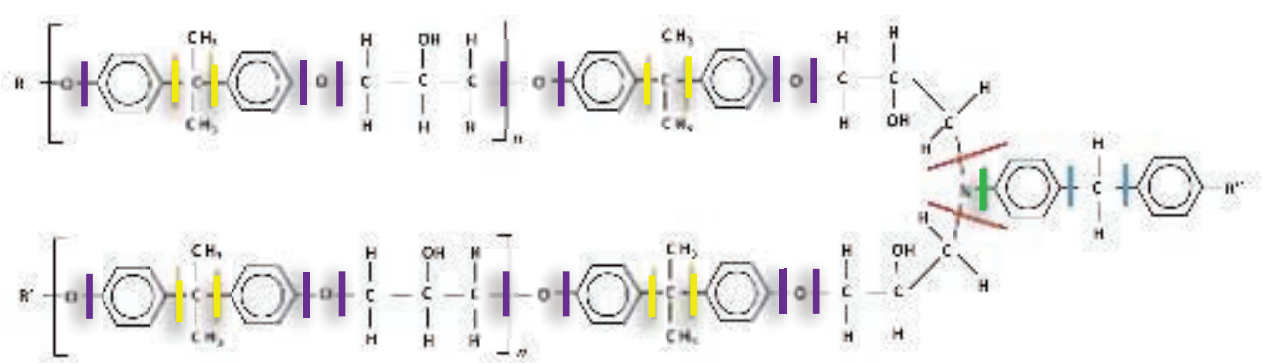

a)

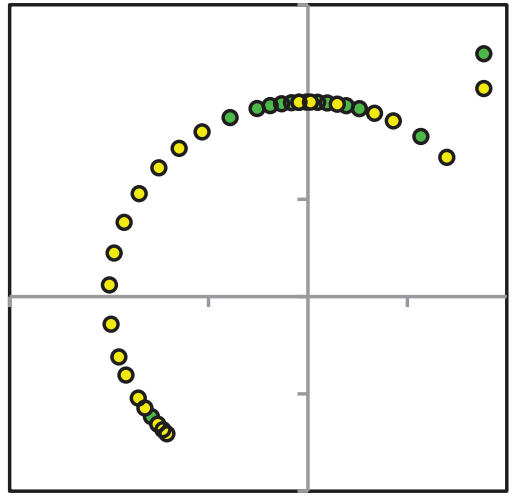

b)

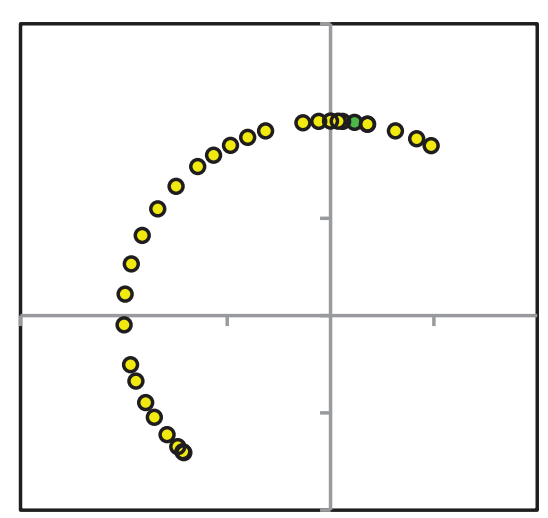

c)

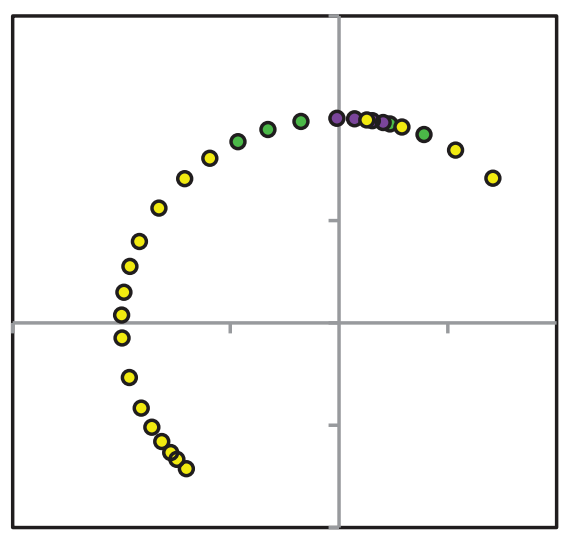

d)

Figure 22. Bond break type results. Shown are the color-coding of bond types (a) and polar plots showing rupture bond type for systems n0 (b), n3 (c), and n5 (d). Arbitrary units.

Because inter-atomic interactions are directly represented here, the results can be compared with fracture theories for polymers which include explicit treatment of atomic and molecular phenomena. Two of the most well-known are those of Zhurkhov [82] and Peterlin [83]. In Zhurkhov's model, the bond scission rate $K_{b}$ becomes biased by the presence of tensile stress. That is

$$
K_{b}=K_{f} \exp [-(\Delta E-C \sigma) / k T]
$$


where $K_{f}$ is the molecular oscillation frequency and $\Delta E$ represents the size of the energy barrier to break a bond in an unstressed system. $C \sigma$ represents the bias to activation due to the presence of tensile stress. It is also indicative of the energy associated with stress concentration local to the bond. While experiments have identified mechano-radicals formed in thermoplastics under deformation [81], information about the mechanical energetic state local to radicals as they formed wasn't inferred from the measurements.

While the simulations presented here don't include scission kinetics and the associated energy barrier landscape, the state local to selected atoms for a given applied bulk stress can be examined in detail. The energy associated with potential interactions of a given atom with its neighbors as well as its thermal energy can be estimated. Because the former is associated with forces, associated directions can also be determined. The per-atom contribution tensor can be estimated by [59]

$$
\begin{aligned}
& \hat{E}_{i j}=-\left[m v_{i} v_{j}+\frac{1}{2} \sum_{n=1}^{N_{p}}\left(r_{1 i} F_{1 j}+r_{2 i} F_{2 j}\right)+\frac{1}{2} \sum_{n=1}^{N_{b}}\left(r_{1 i} F_{1 j}+r_{2 i} F_{2 j}\right)\right. \\
& +\frac{1}{3} \sum_{n=1}^{N_{a}}\left(r_{1 i} F_{1 j}+r_{2 i} F_{2 j}+r_{3 i} F_{3 j}\right)+\frac{1}{4} \sum_{n=1}^{N_{d}}\left(r_{1 i} F_{1 j}+r_{2 i} F_{2 j}+r_{3 i} F_{3 j}+r_{4 i} F_{4 j}\right) \\
& \left.+\frac{1}{4} \sum_{n=1}^{N_{i}}\left(r_{1 i} F_{1 j}+r_{2 i} F_{2 j}+r_{3 i} F_{3 j}+r_{4 i} F_{4 j}\right)+\operatorname{Kspace}\left(r_{k i}, F_{k j}\right)+\sum_{n=1}^{N_{f}}\left(r_{k i} F_{k j}\right)\right]
\end{aligned}
$$

where $i$ and $j$ denote directional indices (i.e., $x, y, z), r$ is particle location, and $F$ is force. The successive terms correspond with kinetic energy, pair, bond, angle, dihedral, improper, kspace, and fix interactions, respectively. Each summation is performed over the number of interactions of the given type in which the atom participates. For terms associated with multi-atom interactions, the associated energy is apportioned according to the number of interacting atoms. Summing the terms in $\hat{E}_{i j}$ for all atoms over the cell volume would result in the stress tensor of the ensemble. Because bond energies are tracked on-the-fly, the state of each atom in the system can be captured when a bond rupture is imminent.

$\hat{E}_{i j}$ results were examined by expressing them as two scalars which contribute to the computation of invariants of the bulk stress tensor. These are

$$
\hat{E}_{1}=\left(\hat{E}_{x x}+\hat{E}_{y y}+\hat{E}_{z z}\right)
$$

and

$$
\hat{E}_{\text {eff ective }}=\sqrt{\frac{\left(\hat{E}_{x x}-\hat{E}_{y y}\right)+\left(\hat{E}_{y y}-\hat{E}_{z z}\right)+\left(\hat{E}_{z z}-\hat{E}_{x x}\right)+6\left(\hat{E}_{x y}{ }^{2}+\hat{E}_{y z}{ }^{2}+\hat{E}_{z x}{ }^{2}\right)}{2}}
$$

Analogous to the earlier discussion, $\hat{E}_{1}$, is associated with dilatational energy present in the vicinity of the atom. $\hat{E}_{\text {effectiv }}$ is associated with local shear energy. 
Investigation focused on the energy for atom types which were found most frequently in highlystrained bonds. Further, there were two atom types within these bonds which were not found elsewhere in the molecular structure. These are nitrogen at crosslinks and aliphatic carbon in the backbone (shown circled in Figure 23. The aliphatic interphenyl backbone carbon and crosslink nitrogen atoms investigated.). $\hat{E}$ data was generated for system $\mathrm{n} 0$ for five load cases, biaxial tension, tension, shear, compression, and biaxial compression. In all simulations, stress was applied in the $x-y$ plane. The correspondence among load cases and the signs of the respective stresses applied is given in Table 3 .
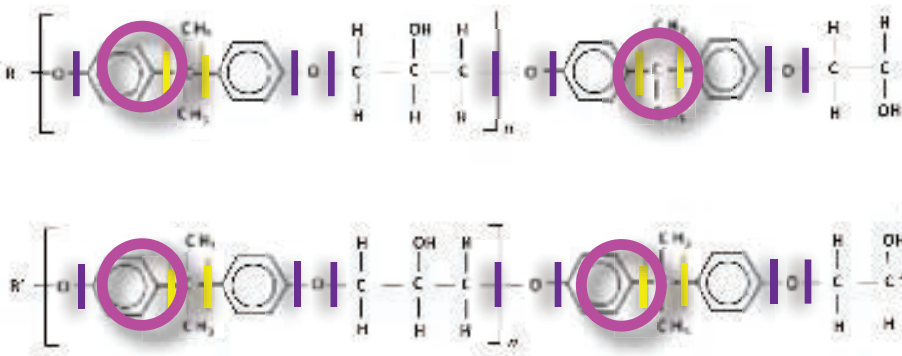

Figure 23. The aliphatic interphenyl backbone carbon and crosslink nitrogen atoms investigated.

Table 3. Signs of the stresses applied for various load cases.

Peterlin's theory [83] anticipated that, in tension or shear, various molecular strands would carry

\begin{tabular}{|l|c|c|}
\hline Load case & $\boldsymbol{x}$ & $\boldsymbol{y}$ \\
\hline Biaxial tension & + & + \\
\hline Tension & 0 & + \\
\hline Shear & - & + \\
\hline Compression & - & 0 \\
\hline $\begin{array}{l}\text { Biaxial } \\
\text { compression }\end{array}$ & - & - \\
\hline
\end{tabular}

663 differing portions of the applied load with strands having a shorter contour length generally carrying a larger proportion. In order to investigate the geometric ordering of loaded atoms according to strands or other geometric entities, atoms were color coded according $\hat{E}_{1}$ and $\hat{E}_{\text {effectiv }}$ levels and displayed by location. $\hat{E}_{1}$ results are shown in Figure 25 through Figure 29. $\hat{E}_{\text {eff ectiv }}$ results are shown in

Figure 30 through Figure 34. All figures depict the cell just prior to first bond rupture.

There are two general features associated with the formation of free volume pockets (i.e., nanoscale voids). First, approximately spherical voids formed in both tension cases in numerous locations. In shear, there were fewer voids which tended to be elongated in the direction of positive stress. Consistent with the negative $J_{1}$ values presented earlier, compression cases did

672 not show voids. These results are also consistent with those of other researchers who found 673 porosity generated in first-quadrant loading. 
674 Second, atoms at void surfaces did not tend to be at elevated levels of $\hat{E}_{1}$ or $\hat{E}_{\text {effectiv }}$. This 675 indicates that (unlike the macroscale continuum analog) the presence of concavity in the 676 assembly of atoms did not correlate with a concentration of deformational energy. Rather, it 677 appears these form when adjacent backbone strands move away from each other and that 678 loading redistribution is accommodated by segmental motions.

679 Consistent with Peterlin, in tensile cases, both atom types along a single strand reached high 680 positive $\hat{E}_{1}$ and $\hat{E}_{\text {effectiv }}$ levels. The most highly-strained bond occurred along these strands. In 681 shear, it appears there are multiple highly-strained strands. In compression, there is no 682 apparent spatial order to the high- $\hat{E}$ atoms and they are dispersed across the cell. The 683 associated strength in the tension cases is therefore sensitive to the bond interactions among a 684 small portion of the population. This apparent sensitivity is also consistent with results of 685 experiments in which fracture occurs in tension cases whereas ductile flow occurs in 686 compression.

687

688 There are several features of the $\hat{E}_{\text {effectiv }}$ results. Crosslinked nitrogen atoms are generally at 689 higher $\hat{E}_{\text {effectiv }}$ than the aliphatic backbone carbon. Unlike the carbons, these nitrogens are 690 located where load is transmitted across junctures among molecular strands. In compression 691 cases, there is more variegation in $\hat{E}_{\text {effectiv }}$ among the carbon atoms than there is among the 692 nitrogens. This lack of good distribution of strain energy among the carbons may be associated 693 with the higher likelihood of carbon-carbon bond reaching critical values (as shown in Figure 694 22). Rather than generally decreasing $\hat{E}_{1}$ levels, compression led to higher levels of $\hat{E}_{\text {eff ectiv }}$. 695 Because plane stress is being applied, the shearing energy indicated by elevated $\hat{E}_{\text {effectiv }}$ may be 696 associated with the bulk shearing strain due to differences among out-of-plane and in-plane 697 strains. 

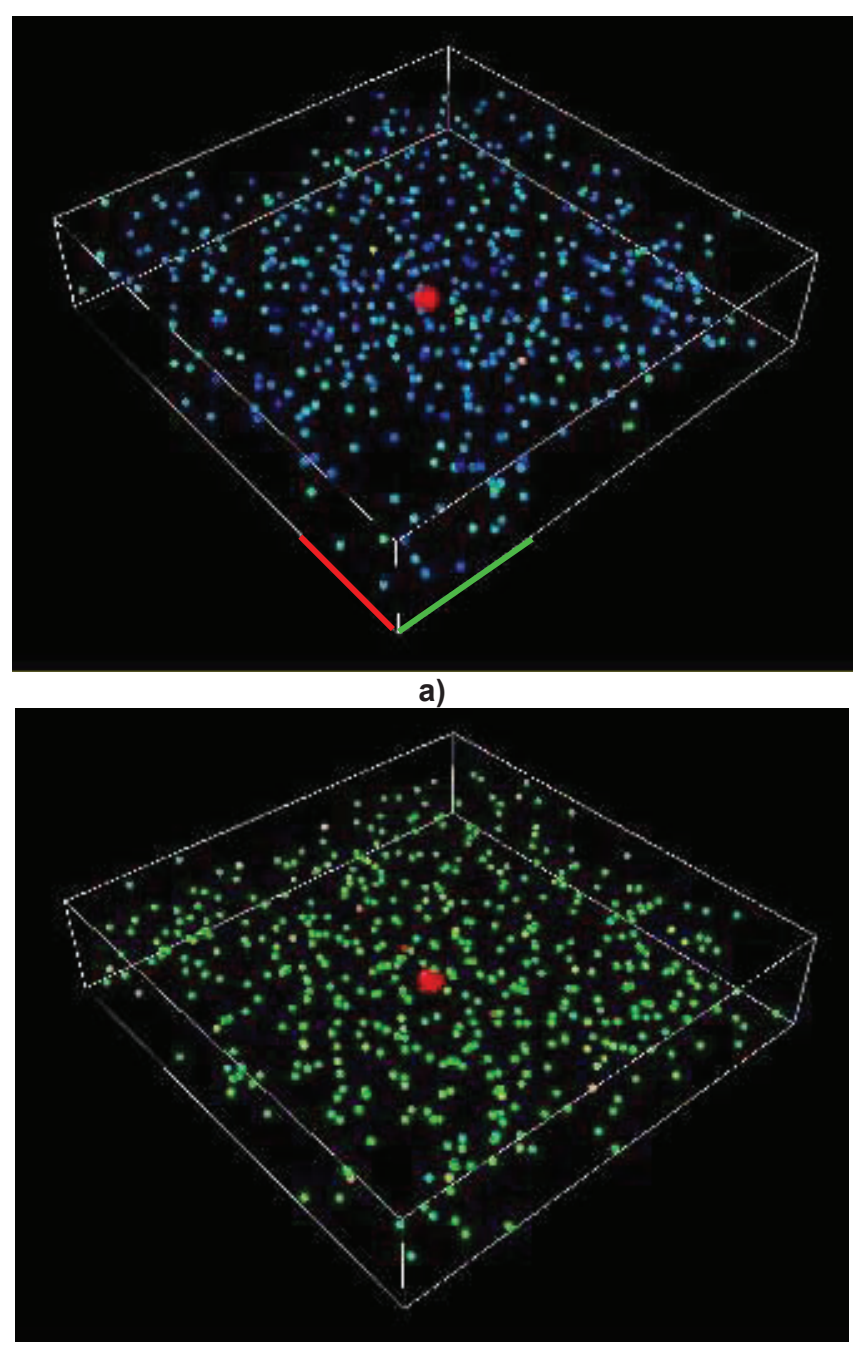

701

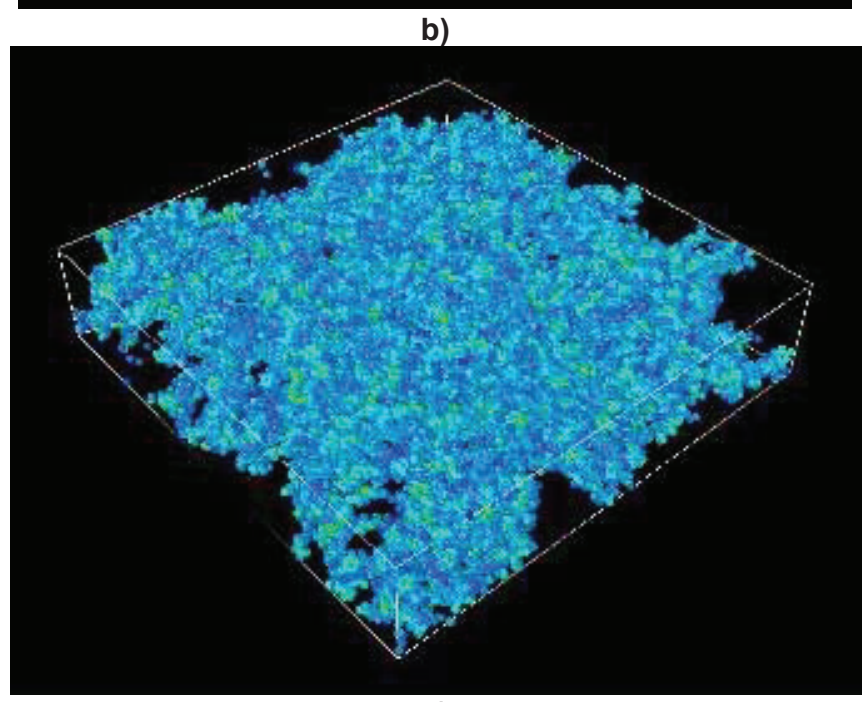

c)

705 Figure 25. Atom locations at incipient rupture in biaxial tension. Atoms are colored according to $\hat{E}_{1}$. Colors 


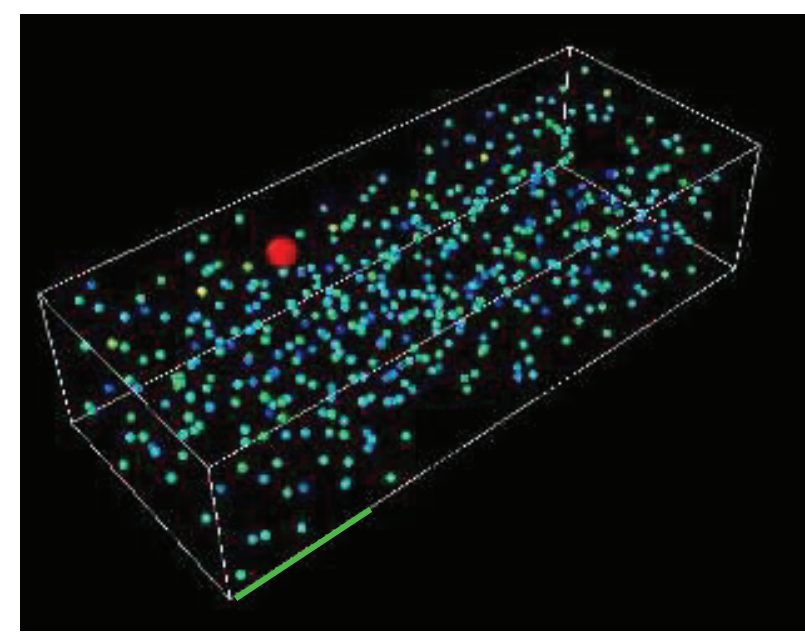

a)

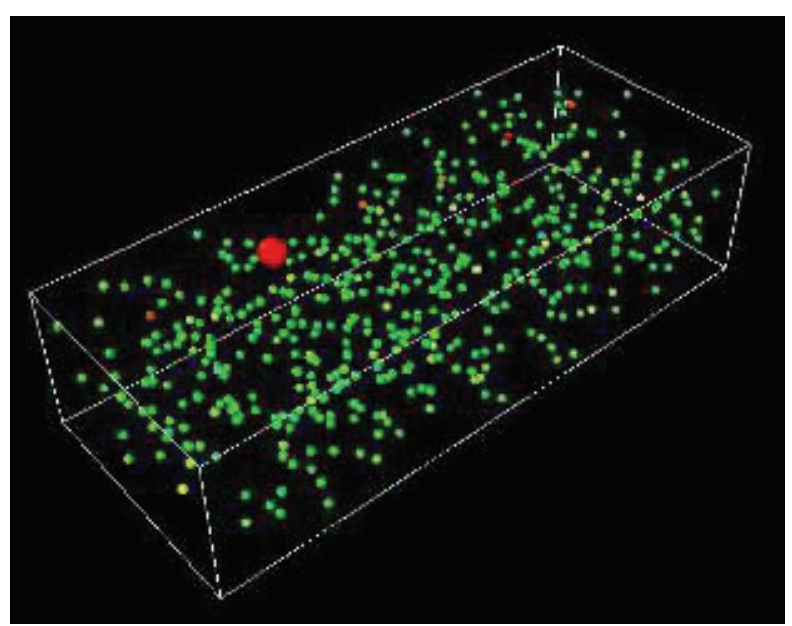

b)

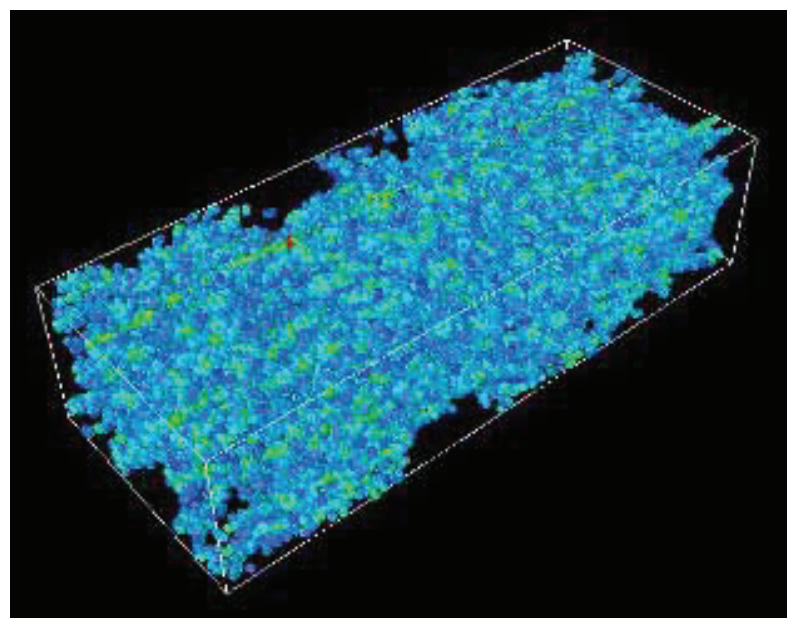

c)

Figure 25. Atom locations at incipient rupture in uniaxial tension. Atoms are colored Figure 25. Stress is applied in the y direction (denoted by the green mark). 


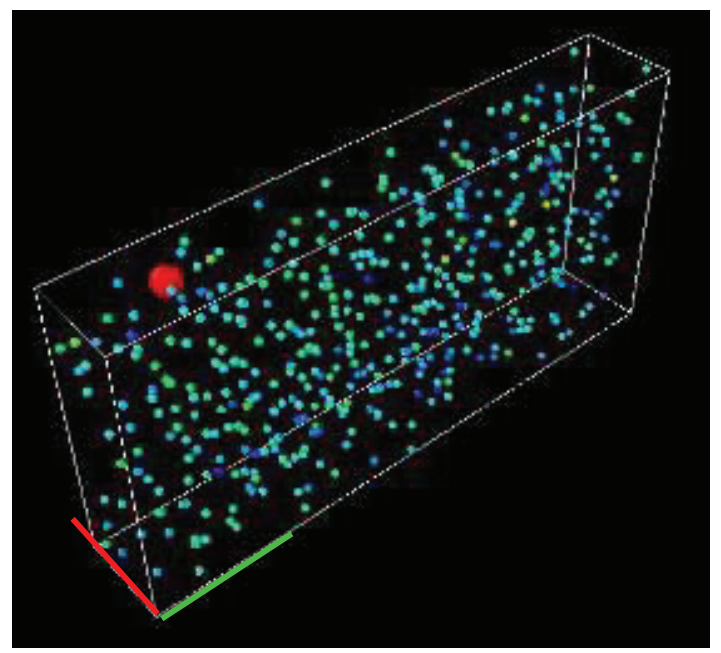

a)

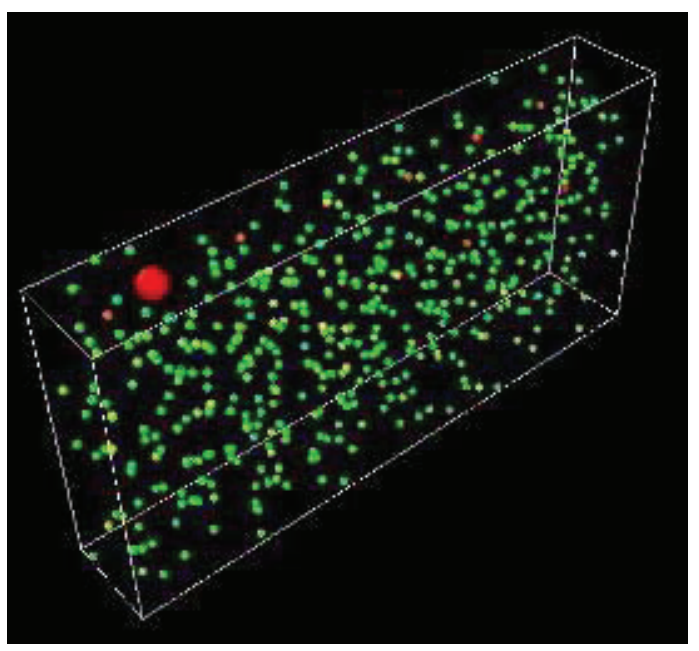

b)

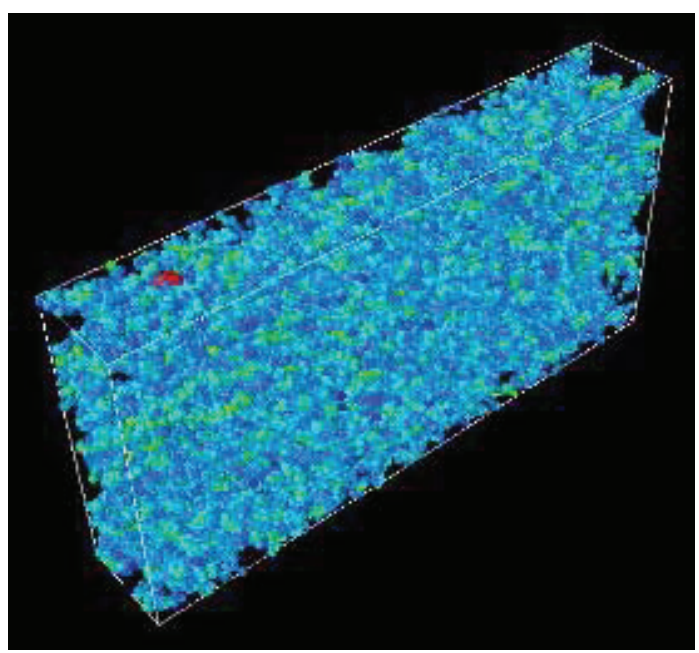

c)

Figure 26. Atom locations at incipient rupture in shear. Atoms are colored according to $\hat{E}_{1}$. The color scaling, atoms depicted, and strained-bond mark are the same as Figure 25. Stresses are applied in the $x$ and $y$ directions (denoted by the red and green marks, respectively). 


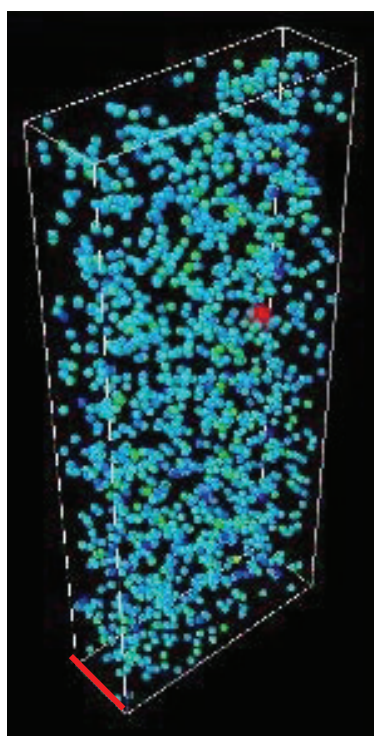

a)

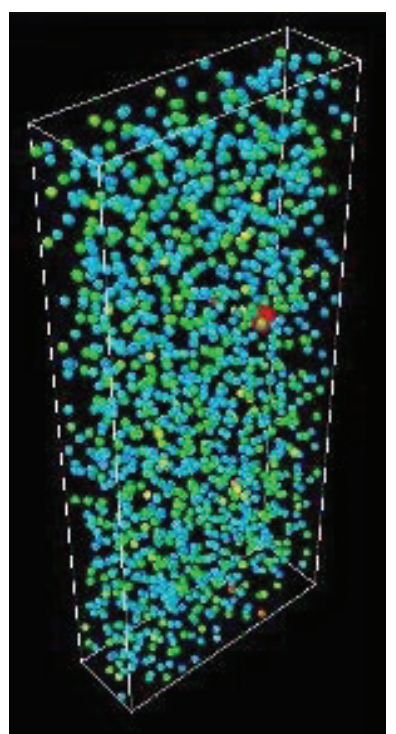

b)

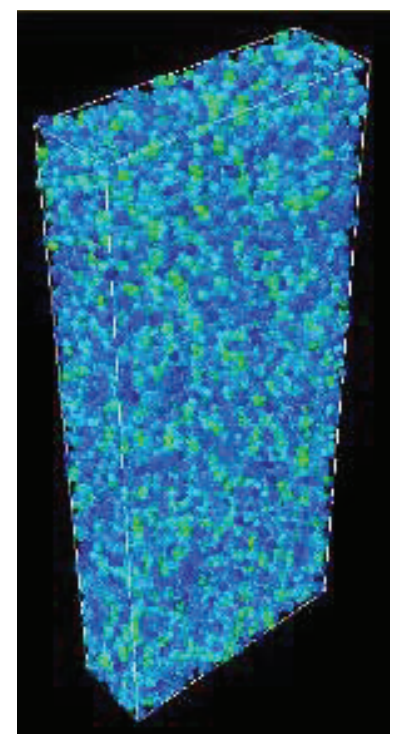

c)

Figure 27. Atom locations at incipient rupture in compression. Atoms are colored according

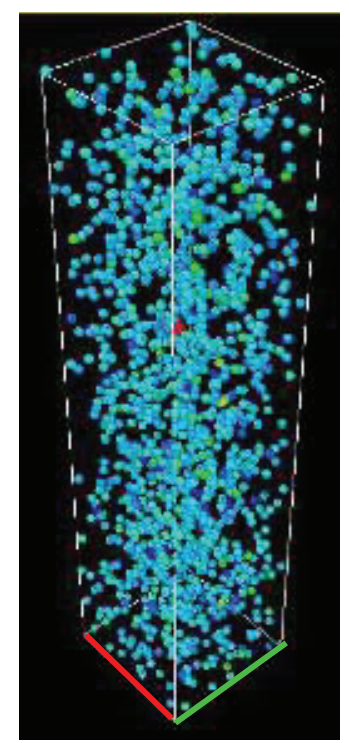

a)

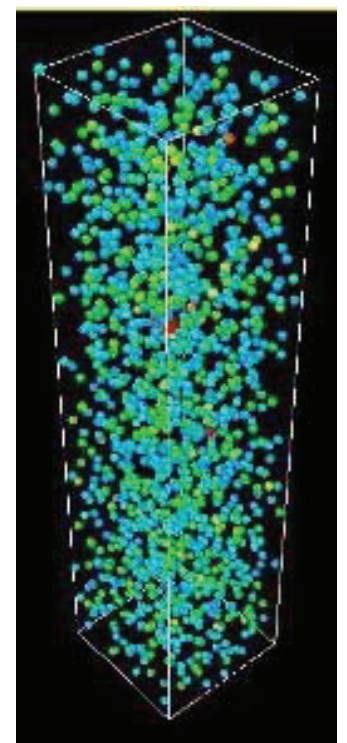

b)

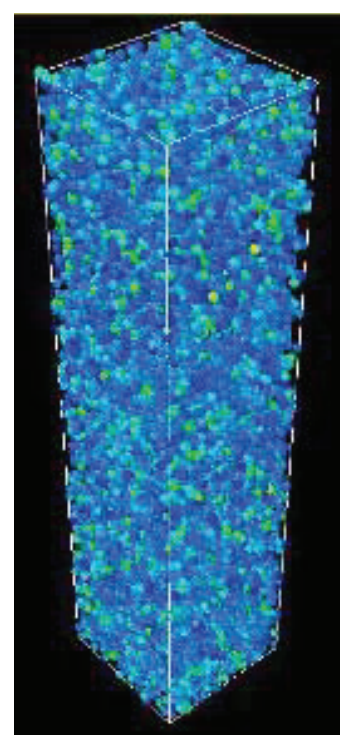

c)

Figure 29. Atom locations at incipient rupture in biaxial compression. Atoms are colored 


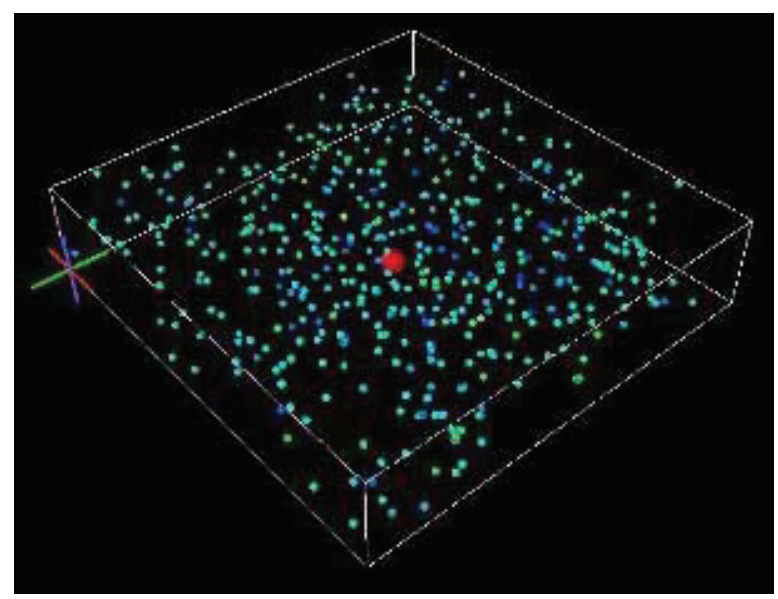

a)

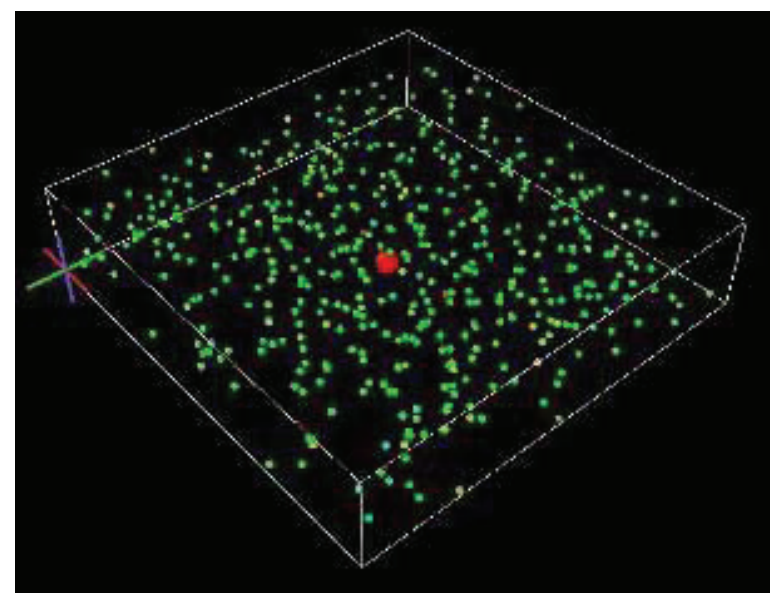

b)

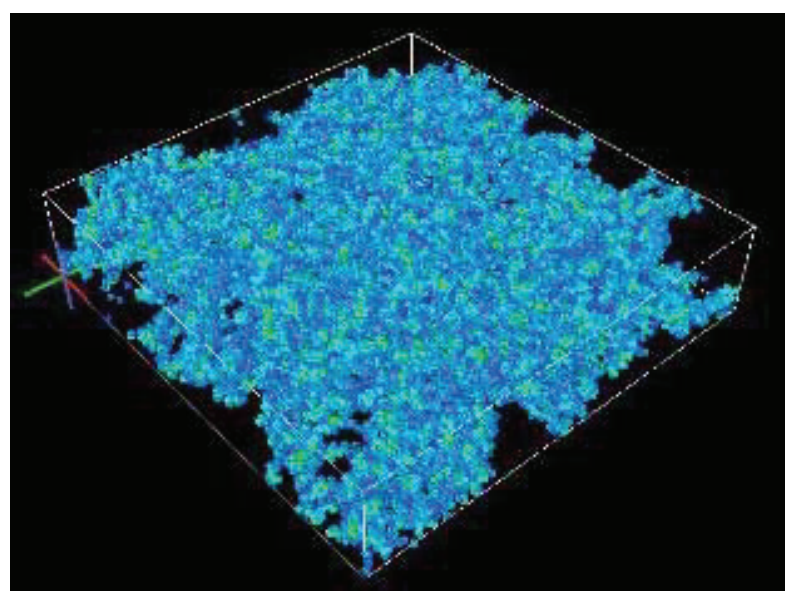

c)

Figure 30. Atom locations at incipient rupture in biaxial tension. Atoms are colored according to $\hat{E}_{\text {eff ectiv }}$. The color scale ranges from 0.0 (dark blue) to $396 \mathrm{kcal} / \mathrm{mol}$ (red). Shown are cross-linked nitrogen (a), inter-phenyl backbone carbon (b), and all atoms (c). $x$ and $y$ directions are denoted by red and green marks, respectively. 


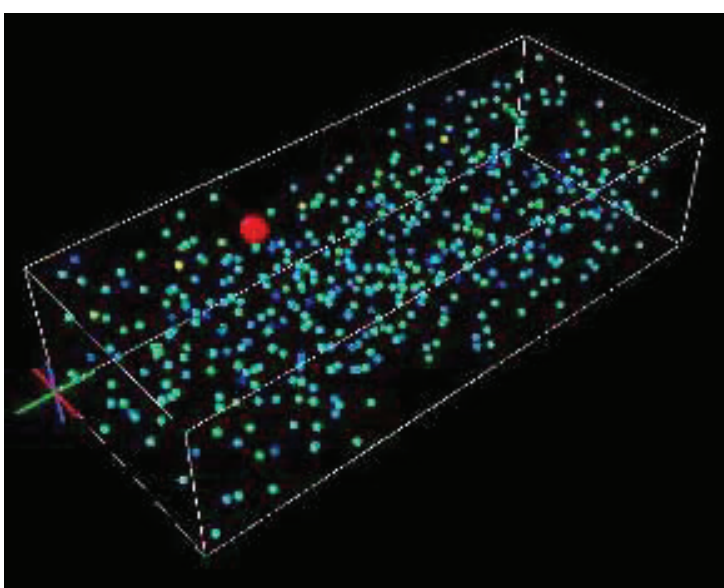

a)

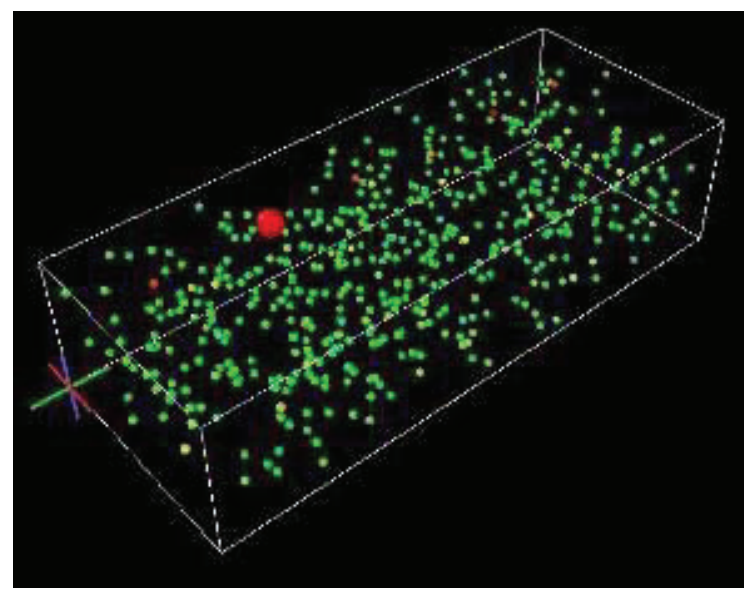

b)

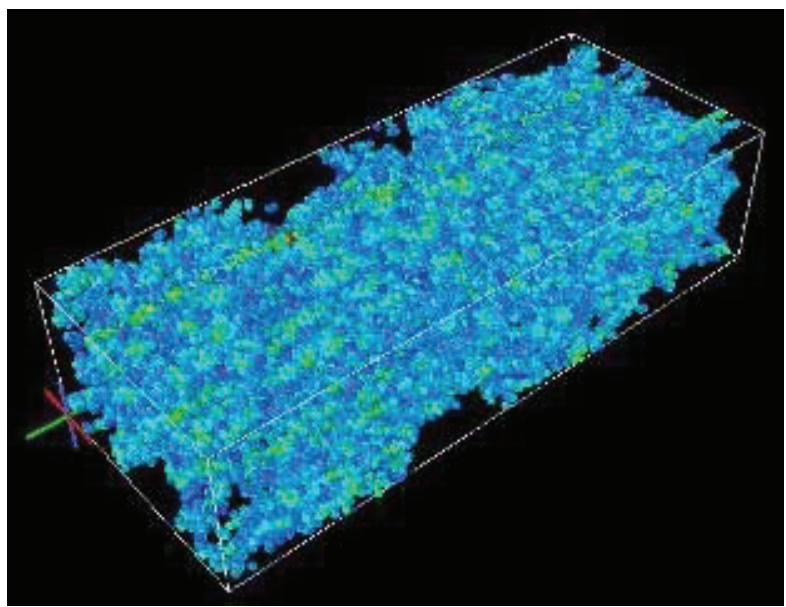

c)

756

Figure 30. Atom locations at incipient rupture in uniaxial tension. Atoms are colored according to $\hat{E}_{e f f e c t i v}$. The color scaling, atoms depicted, axes, and strained-bond mark are the same as Figure 30. 


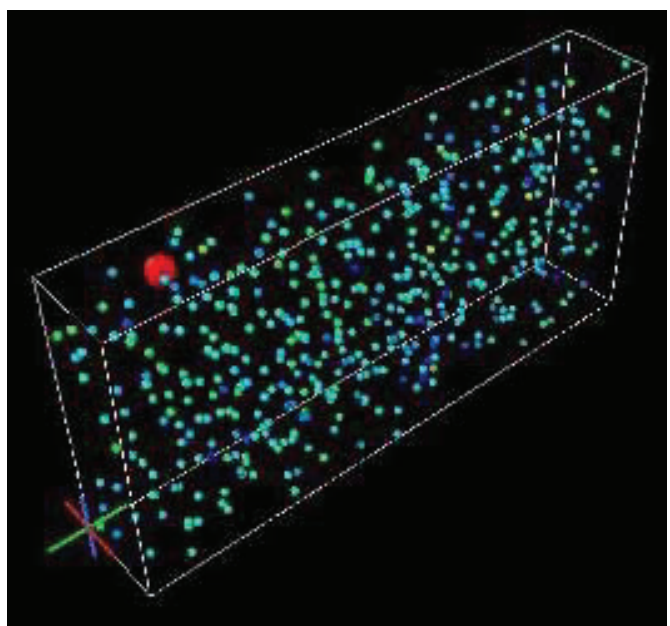

a)

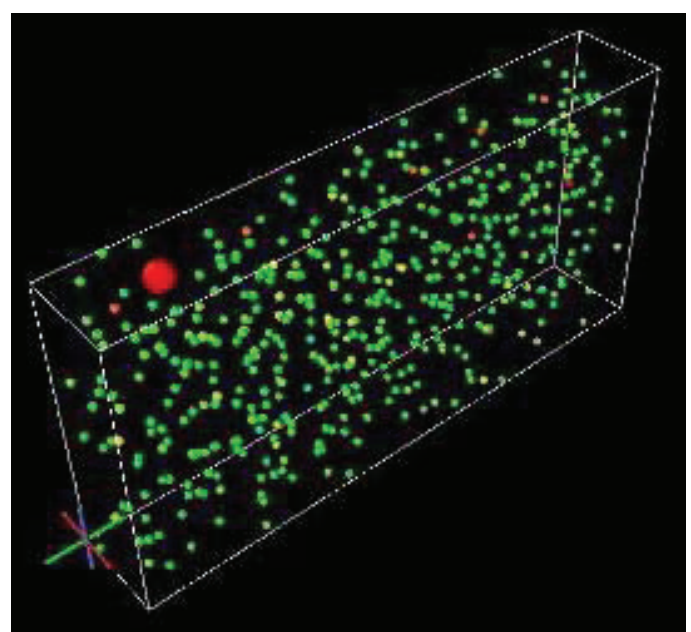

b)

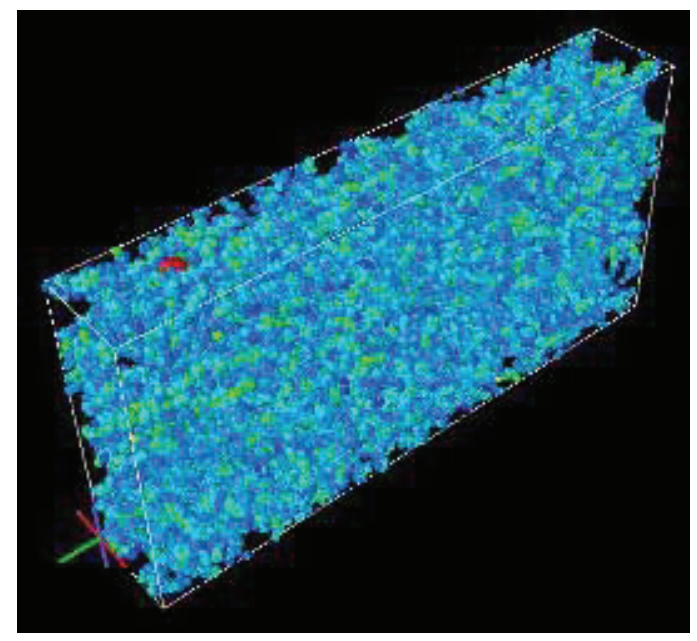

c)

763

764

765

766

Figure 31. Atom locations at incipient rupture in shear. Atoms are colored according to $\hat{E}_{\text {effectiv }}$. The color scaling, atoms depicted, axes, and strained-bond mark are the same as Figure 30. 


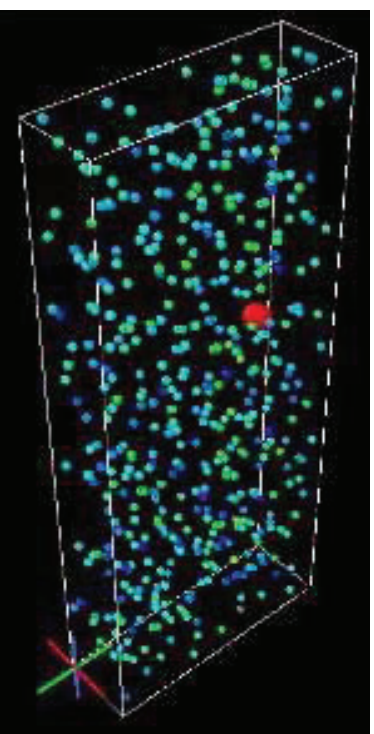

a)

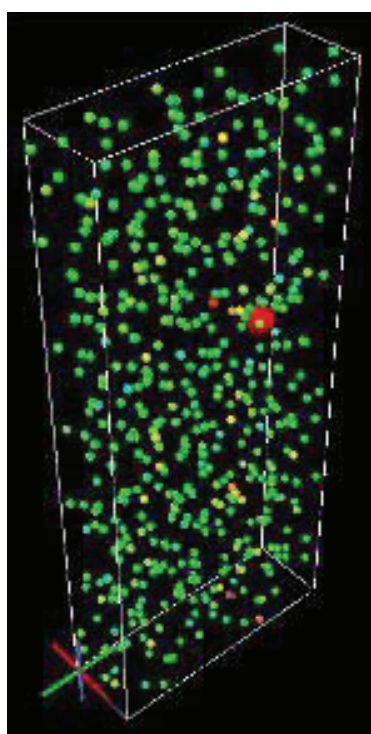

b)

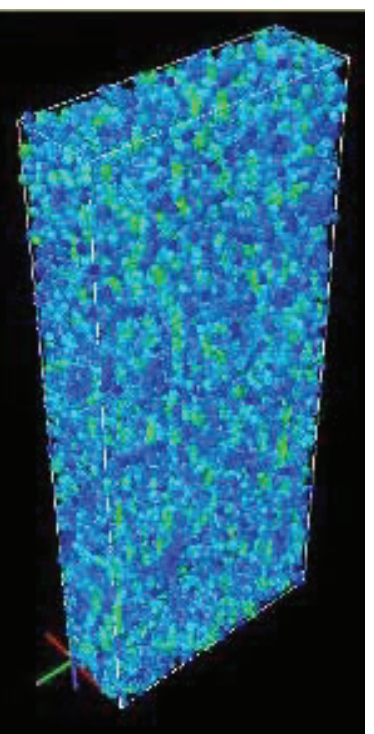

c)
769

770

Figure 32. Atom locations at incipient rupture in uniaxial compression. Atoms are colored according to $\hat{E}_{\text {ef } f \text { ectiv }}$. The color scaling, atoms depicted, axes, and strained-bond mark are the same as Figure 30.

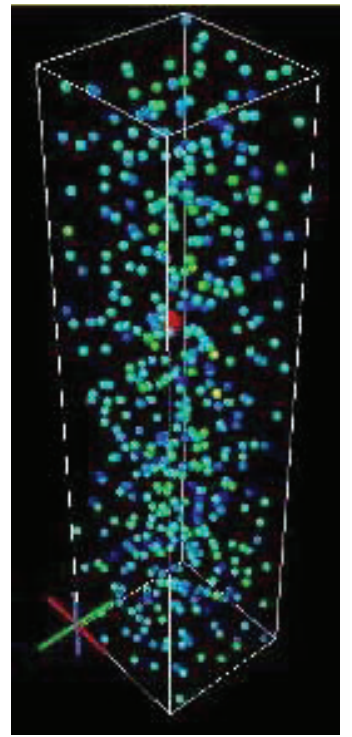

a)

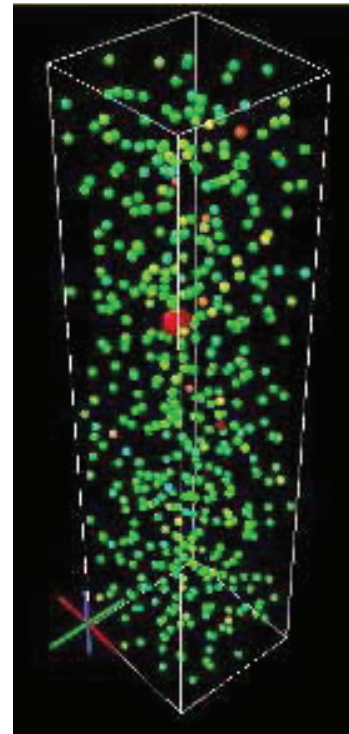

b)

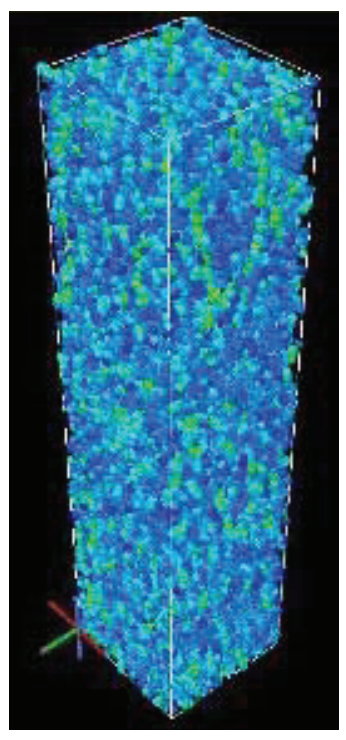

c)

Figure 34. Atom locations at incipient rupture in biaxial compression. Atoms are colored according to $\hat{E}_{e f f e c t i v}$. The color scaling, atoms depicted, axes, and strained-bond mark are the same as Figure 30. 


\section{Conclusions}

781 An algorithm and results for atomistic simulation of generalized plane stress in glassy 782 thermosets is presented. This simulation approach is realistic in that stresses are modulated to 783 attain a target strain rate.

784 Cured epoxy systems scaled to include at least 100 crosslink sites have been investigated. The predicted elastic and strength properties show good agreement with measured values. The system response in plane stress shows agreement with continuum yield theories commonly applied to polymers. The evidence also supports the findings from experiments that strength has a different functional dependency in first-quadrant loading compared with the second and third. The results here show that plastic behavior, the development of nano-scale porosity, large increases in van der Waals energy after yield, and small changes in molecular torsional energy are differentiating features of the first-quadrant loading.

The rupture results provide information that complements the information provided by experiments of others and allows for testing of theories. Differing weak points in the crosslinked molecular structure according to load case are identified. The environment local to covalent bonds can be described. The geometric arrangements of atoms at high energy are consistent with molecular theories of molecular structural rupture in tension and provide insight into the geometric arrangements which may occur in other loading cases.

\section{Acknowledgements}

799 This work was supported by U.S. Air Force Office of Scientific Research, Project \#: 800 14RX09COR. "Multiscale Mechanics of Bond Scission and Interfacial Properties of Polymer 801 Composites". The authors would also like to thank the Department of Defense High 802 Performance Computing Modernization Program for computer time as well as the User 803 Productivity Enhancement Technology Transfer and Training program for support.

\section{Works Cited}

[1] D. Drucker and W. Prager, "Soil mechanics and plastic analysis for limit design," Quarterly of Applied Mathematics, vol. 10, no. 2, pp. 157-165, 1952.

[2] R. v. Mises, "Mechanik der festen Korper im plastisch-deformalen Zustand," Gottinger Nachr., math.-phys., pp. 582-592, 1913.

[3] H. Hencky, "Uber die statisch bestimmte Falle des Gleichwichts in plastischen Korpern," Zeitschr. angew. Mathematik u. Mechanik, vol. 3, pp. 241-251, 1923. 
[4] W. Burzynski, "Uber die Anstrengungshypotheses," Schweizerische Bauzeitung, vol. 94, pp. 259-262, 1929.

[5] Y. Yagn, "New methods of strength prediction," Vestnik inzhenerov i tekhnikov, vol. 6, pp. 237-244, 1931.

[6] H. Tresca, "Mémoire sur l'écoulement des corps solides soumis à de fortes pressions," C.R. Acad. Sci. Paris, vol. 59, p. 754 ff, 1864.

[7] C. A. Coulomb, "Essai sur une application des regles des maximis et minimis a quelquels problemesde statique relatifs, a la architecture.," Mem. Acad. Roy. Div. Sav., vol. 7, pp. 343-387, 1776.

[8] R. Kody and A. Lesser, "Deformation and yield of epoxy networks in constrained states of stress," Journal of Materials Science, vol. 32, pp. 5637-5643, 1997.

[9] J. Sultan and F. McGarry, "Effect of Rubber Particle Size on Deformation Mechanisms in Glassy Epoxy," Polymer Engineering and Science, vol. 13, no. 1, pp. 29-34, 1975.

[10] J. Gosse, "Strain invariant failure criteria for polymers in composite materials," in AIAA/ASME/ASCE/AHS/ASC Structures, Structural Dynamics, and Materials Conference, Seattle, 2011.

[11] S. Sternstein and L. Ongchin, "Yield criteria for plastic deformation of glassy high polymers in general stress fields," in ACS Polymer Preprints, 1969.

[12] L. Asp, L. Berglund and R. Talreja, "A criterion for crack initiation in glassy polymers subjected to a composite-like stress state," Composites Science and Technology, vol. 56, pp. 1291-1301, 1996.

[13] A. Argon, "Physical Basis of Distortional and Dilational Plastic Flow in Glassy Polymers," J. Macromol. Sci.-Phys. B, vol. 8, no. 3-4, pp. 573-596, 1973.

[14] R. Raghava, R. Caddell and G. Yeh, "The macroscopic yield behavior of polymers," J. Mater. Sci. , vol. 8, pp. 225-232, 1973.

[15] J. Bauwens, "Yield condition and propagation of Luders lines in tension-torsion experiments on poly(vinyl chloride)," J. Polym. Sci. A, vol. 8, pp. 893-901, 1970.

[16] P. Bowden and J. Jukes, "The plastic flow of isotropic polymers," J. Mater. Sci. , vol. 7, pp. 52-63, 1972.

[17] I. Ward, "Review: The yield behavior of polymers," J. Mater. Sci. , vol. 6, pp. 1397-1417, 
1971.

[18] A. Smith, S. Wilkinson and W. Reynolds, "The elastic constants of some epoxy resins," J. Mat/s. Sci. , vol. 9, pp. 547-550, 1974.

[19] I. L. A. Yannas, "Inter- versus intramolecular energy barriers," Polym. Prepr., vol. 16, pp. 564-569, 1975.

[20] R. Robertson, "Theory for the Plasticity of Glassy Polymers," J. Chem. Phys., vol. 44, pp. 3950-3956, 1966.

[21] P. Bowden and R. Raha, "A molecular model for yield and flow in glassy amorphous polymers making use of a dislocation analogue," Philosophical Magazine, vol. 29, pp. 149166, 1974.

[22] S. Joseph, "Cooperative model for the mechanical deformation of polymers," J. Polym. Sci.: Polym. Phys. Ed. , vol. 16, no. 6, pp. 1071-1084, 1978.

[23] A. Argon, "A theory for the low-temperature plastic deformation of glassy polymers," Philosophical Magazine, vol. 28, no. 4, pp. 839-865, 1973.

[24] A. Argon and M. Bessonov, "Plastic Flow in Glassy Polymers," Polymer Engineering and Science, vol. 17, no. 3, pp. 174-182, 1977.

[25] O. Hasan, M. Boyce, X. Li and S. Berko, "An Investigation of the Yield and Postyield Behavior and Corresponding structure of Poly(methyl methacrylate)," J. Polym. Sci. B Polym. Phys., vol. 31, pp. 185-197, 1993.

[26] Y. Jean, Q. Deng and T. Nguyen, "Free-Volume Hole Properties in Thermosetting Plastics Probed by Positron Annihilation Spectroscopy: Chain Extension Chemistry," Macromolecules, vol. 28, pp. 8840-8844, 1995.

[27] G. Dlubek, J. Pointek, M. Shaikh, E. Hassan and R. Krasue-Rehberg, "Free volume of an oligomeric epoxy resin and its relation to structural relaxation: Evidence from positron lifetime and pressure-volume-temperature experiments," Phys. Rev. E, vol. 75, p. 021802 ff, 2007.

[28] M. Debowska, L. Kurzeja, A. Baranowski, K. Hennek, K. Jerie and J. Rudzinska-Girulska, "Influence of structure of crosslinked epoxy resin on its properties studied by positron annihilation and other methods," J. Radioanalytical and Nuclear Chemistry, vol. 210, pp. 485-494, 1996.

[29] S. Goyanes, G. Rubiolo, W. Salgueiro and A. Somoza, "On the free volume evolution in a deformed epoxy composite. A positron annihilation study," Polymer, vol. 46, pp. 9081-9087, 
2005.

[30] J. Ramos, E. Serrano, A. Tercjak, W. Salgueiro, S. Goyanes and I. Mondragon, "PALS study of epoxy matrices: self-assembly of block copolymers and its capability for nanostructuring thermosetting systems," Phys. Stat. Sol., vol. 4, pp. 3690-3699, 2007.

[31] M. Paluch, C. Roland, J. Gapinski and A. Patkowski, "Pressure and temperature dependence of structural relaxation in diglycidylether of bisphenol A," J. Chem Phys., vol. 118, pp. 3177-3186, 2003.

[32] T. Scherzer, "Characterization of the Molecular Deformation Behavior of Glassy Epoxy Resins by Rheo-optical FTIR Spectroscopy," J. Polym. Sci. B Polym. Phys., vol. 34, pp. 459-470, 1996.

[33] T. Scherzer, "Rheo-optical fourier transform infrared spectroscopy of diol-modified diglycidyl ether of bisphenol-A epoxy resins," Polym. Bulletin, vol. 42, pp. 579-586, 1999.

[34] S. Sikka and H. Kausch, "Study of stress-induced changes in poly(ethylene terephthalate) through Fourier transform infrared spectroscopy," Coll. Polym. Sci., vol. 257, pp. 10601067, 1979.

[35] B. Fanconi, K. DeVries and R. Smith, "Free radicals and new end groups resulting from chain scission: 2. Mechanical degradation of polyethylene," Polymer, vol. 23, pp. 10271033, 1982.

[36] S. Zhurkov, V. Zakrevskyi, V. Korsukov and V. Kuksenko, "Mechanism of Submicrocrack Generation in Stressed Polymers," J. Polym. Sci. A-2, vol. 10, pp. 1509-1520, 1972.

[37] B. Fanconi, "Chain scission and mechanical failure of polyethylene," J. Appl. Phys., vol. 54, pp. 5577-5582, 1983.

[38] H. Kausch-Blecken von Schmeling and J. Becht, "Über Spannungsrelaxation und zeitabhängige elastische Bruchvorgänge in orientierten Fasern," Rheol. Acta, vol. 9, pp. 137-144, 1970.

[39] F. Capaldi, M. Boyce and G. Rutledge, "Molecular response of a glassy polymer to active deformation," Polymer, vol. 45, pp. 1391-1399, 2004.

[40] D. Brown and J. Clarke, "Molecular Dynamics Simulation of an Amorphous Polymer under Tension. I. Phenomenology," Macromolecules, vol. 24, pp. 2075-2082, 1991.

[41] J. Bouvard, D. Ward, D. Hossain, E. Marin, D. Bammann and M. Horstmeyer, "A general inelastic internal state variable model for amorphous glassy polymers," Acta Mechanica, 
vol. 213, pp. 71-96, 2010.

[42] M. Buehler, A. Hartmaier, M. Duchaineau, F. Abraham and H. Gao, "The dynamical complexity of work-hardening: a large-scale molecular dynamics simulation," Acta Mech. Sinica, vol. 21, pp. 103-111, 2005.

[43] Y. Han and J. Elliot, "Molecular dynamics simulations of the elastic properties of polymer/carbon nanotube composites," Comp. Matl. Sci., vol. 39, pp. 315-323, 2007.

[44] A. Awasthi, D. Lagoudas and D. Hammerand, "Modeling of graphene-polymer interfacial mechanical behavior using molecular dynamics," Modelling Simul. Mater. Sci. Eng., vol. 17, p. 015002 ff, 2009.

[45] G. Odegard, B. Jensen, S. Gowtham, J. Wu, J. He and Z. Zhang, "Predicting Mechanical Response of Crosslinked Epoxy using ReaxFF," Chem. Phys. Lett., vol. 591, pp. 175-178, 2014.

[46] B. Jensen, K. Wise and G. Odegard, "The Sensitivity of ReaxFF Simulations to Tensile Failure of Diamond, Graphene, and Carbon Nanotube to Simulation Parameters," J. Comp. Chem., vol. 36, pp. 1587-1596, 2015.

[47] D. Hossain, M. Tschopp, D. Ward, J. Bouvard, P. Wang and M. Horstemeyer, "Molecular dynamics simulations of deformation mechanisms of amorphous polyethylene," Polymer, vol. 51, pp. 6071-6083, 2010.

[48] J. Moller, S. Barr, E. Schultz, T. Breitzman and R. Berry, "Simulation of fracture nucleation in cross-linked polymer netowrks," JOM, vol. 65, pp. 147-167, 2013.

[49] S. Christensen and R. D'Oyen, "Computational formulation of a new composite matrix," Scripta Materialia, vol. 70, pp. 18-24, 2014.

[50] R. Zhu, E. Pan and A. Roy, "Molecular dynamics study of the stress-strain behavior of carbon-nanotube Epon 862 composites," Mat/s. Sci. Engrg. A, vol. 447, pp. 51-57, 2007.

[51] D. Rottach, J. Curro, J. Budzien and G. Grest, "Molecular Dynamics Simulations of Polymer Networks Undergoing," Macromolecules, vol. 40, pp. 131-139, 2007.

[52] V. Sundararaghavan and A. Kumar, "Molecular dynamics simulations of compressive yielding," Intl. J. Plas., vol. 47, pp. 111-125, 2013.

[53] C. Li, A. Browning, S. Christensen and A. Strachan, "Atomistic simulations on multilayer graphene reinforced epoxy composites," Composites A, vol. 43, pp. 1293-1300, 2012. 
[54] E. Jaramillo, N. Wilson, S. Christensen, J. Gosse and A. Strachan, "Energy-based yield criterion for PMMA from large-scale molecular dynamics simulations," Phys. Rev. B, vol. 85, p. 024114 ff, 2012.

[55] C. Li, G. Medvedev, E. Lee, J. Kim and J. Caruthers, "Molecular dynamics simulations and experimental studies of the thermomechan ical response of an epoxy thermoset polymer," Polymer, vol. 53, pp. 4222-4230, 2012.

[56] C. Li, E. Jaramillo and A. Strachan, "Molecular dynamics simulations on cyclic deformation of an epoxy thermoset," Polymer, vol. 54, pp. 881-890, 2013.

[57] C. Schuh and A. Lund, "Atomistic basis for the plastic yield crierion of metallic glass," Nature Materials, vol. 2, pp. 449-452, 2003.

[58] N. Vu-Bac, M. Bessa, T. Rabczuk and W. Liu, "A Multiscale Model for the Quasi-Static Thermo-Plastic Behavior of Highly Cross-Linked Glassy Polymers," Macromolecules, vol. 48, pp. 6713-6723, 2015.

[59] A. Thompson, S. Plimpton and W. Mattson, "General formulation of pressure and stress tensor for arbitrary many-body interaction potentails under periodic boundary conditions," Journal of Chemical Physics, vol. 131, no. 15, pp. 154107-1 ff, 2009.

[60] M. Glad and E. Kramer, "Microdeformation and network structure in epoxies," J. Mat/s. Sci., vol. 26, pp. 2273-2286, 1991.

[61] J. Racich and J. Koutsky, "Nodular Structure in Epoxy Resins," J. Appl. Polym. Sci., vol. 20, pp. 2111-2129, 1976.

[62] J.-P. Pascault, H. Sautereau, J. Verdu and R. Williams, Thermosetting Polymers, New York: Marcel Dekker, 2002.

[63] J. Lemay, B. Swetlin and F. Kelley, "Structure and Fracture of Highly Cross-Linked Networks," in Highly Cross-Linked Polymers, 1983.

[64] A. Charlesby and N. Hancock, "The Effect of Cross-Linking on the Elastic Modulus of Polyethylene," Pro. Roy. Soc. London. Ser. A, vol. 218, pp. 245-255, 1953.

[65] L. LeMay and F. Kelley, "Structure and ultimate properties of epoxy resins. Epoxy Resins and Composites III," Adv. Polym. Sci., vol. 78, pp. 116-148, 1986.

[66] A. Kinloch, C. Finch and S. Hashemi, "Effect of segmental molecular mass between crosslinks of the matrix phase on the toughness of rubber-modified epoxies," Polym. Comm., vol. 28, pp. 322-325, 1987. 
[67] D. Rigby and L. Subramanian, "Contract Research Report for Advanced Composite Polymers Project," Accelrys, March 2009.

[68] G. Levita, S. De Petris, A. Marchetti and A. Lazzeri, "Crosslink density and fracture toughness of epoxy resins," J. Matls. Sci., pp. 2348-2352, 1991.

[69] P. Dauber-Osguthorpe, V. Roberts, D. Osguthorpe, J. Wolff, M. Genest and A. Hagler, "Structure and energetics of ligand binding to proteins: Escherichia coli dihydrofolate reductase-trimethoprim, a drug-receptor system," Proteins, vol. 4, pp. 31-47, 1988.

[70] J. Smook, W. Hamersma and A. Pennings, "The fracture process of ultra-high strength polyethylene fibers," J. Mat/s. Sci. , vol. 19, pp. 1359-1373, 1984.

[71] S. Plimpton, "Fast Parallel Algorithms for Short-Range Molecular Dynamics," J. Comp. Phys. , vol. 117, pp. 1-19, 1995.

[72] R. Gerlach, C. Siviour, N. Petrinic and J. Wiegand, "Experimental characterisation and constitutive modelling," Polymer, vol. 49, pp. 2728-2737, 2008.

[73] Y. Liang and K. Liechti, "On the large deformation and localization behavior of an epoxy resin unde multiaxial stress states," Int. J. Solids Structures, vol. 33, no. 10, pp. 1479-1500, 1996.

[74] W. Knauss and I. Emri, "Volume Change and the Nonlinearly Thermo-Viscoelastic Constitution of Polymers," Polym. Eng. Sci, vol. 27, pp. 86-100, 1987.

[75] A. Charlesby and N. Hancock, "The Effect of Cross-Linking on the Elastic Modulus of Polythene," Proc. Roy. Soc. London A, vol. 218, pp. 245-255, 1953.

[76] J. Littell, C. Ruggeri, R. Goldberg, G. Roberts, W. Arnold and W. Binienda, "Measurement of Epoxy Resin Tension, Compression, and Shear Stress-Strain Curves over a Wide Range of Strain Rates Using Small Test Specimens," Journal of Aerospace Engineering, vol. 21, pp. 162-173, 2008.

[77] D. Kaelble, "The Dynamic Mechanical Properties of Epoxy Resins," SPE Journal, vol. 15, no. 12, pp. 1071-1077, 1959.

[78] D. O'Brien, N. Sotos and S. White, "Cure-dependent Viscoelastic Poison's Ratio of Epoxy," Experimental Mechanics, vol. 47, pp. 237-249, 2007.

[79] Girard-Reydet, V. Vicard, J. Pascault and H. Sauteraeu, "Polyetherimide-modified epoxy networks: Influence of cure conditions on morphology and mechanical properties," J. Appl. Polym. Sci., vol. 65, pp. 2433-2445, 1997. 
[80] A. Mayr, W. Cook and G. Edward, "Yielding behaviou in model epoxy thermostes - I. Effect of strain rate and composition," Polymer, vol. 39, pp. 3719-3724, 1998.

[81] J. Somha, "Mechanochemistry of polymers," Prog. Polym, Sci., vol. 14, pp. 451-596, 1989.

[82] S. Zhurkhov and E. Tomashevsky, "An investigation of fracture process of polymers by electron spin resonance method," Oxford, 1966.

[83] A. Peterlin, "Chain scission and plastic deformation in the strained crystalline polymer," $\mathrm{J}$. Polym. Sci. , Vols. C-32, p. 297 ff., 1971. 